\title{
The Magnus representation and higher-order Alexander invariants for homology cobordisms of surfaces
}

\author{
TAKUYA SAKASAI
}

\begin{abstract}
The set of homology cobordisms from a surface to itself with markings of their boundaries has a natural monoid structure. To investigate the structure of this monoid, we define and study its Magnus representation and Reidemeister torsion invariants by generalizing Kirk, Livingston and Wang's argument over the Gassner representation of string links. Then, by applying Cochran and Harvey's framework of higher-order (noncommutative) Alexander invariants to them, we extract several information about the monoid and related objects.
\end{abstract}

57M05; 57M27, 20F34, 57N05

\section{Introduction}

Let $\Sigma_{g, 1}$ be a compact connected oriented surface of genus $g \geq 1$ with one boundary component. A homology cylinder over $\Sigma_{g, 1}$ consists of a homology cobordism from $\Sigma_{g, 1}$ to itself with markings of its boundary. We denote by $\mathcal{C}_{g, 1}$ the set of isomorphisms classes of homology cylinders. Since stacking two homology cylinders gives a new one, we can endow $\mathcal{C}_{g, 1}$ with a monoid structure (see Section 2 for the precise definition). The origin of homology cylinders goes back to Goussarov [10], Habiro [11], Garoufalidis-Levine [9] and Levine [21], where the clasper (or clover) surgery theory is effectively used to investigate the structure of $\mathcal{C}_{g, 1}$.

As mentioned in [9] and [21], we can construct a homology cylinder from each homology 3-sphere or pure string link. Also, for a given homology cylinder, we can use an element of the mapping class group $\mathcal{M}_{g, 1}$ of $\Sigma_{g, 1}$ to give another one by changing its markings. Since these operations preserve each monoid structure, $\mathcal{C}_{g, 1}$ can be regarded as a simultaneous generalization of the monoid of homology 3-spheres, that of string links and $\mathcal{M}_{g, 1}$, any of which plays an important role in the theory of 3-manifolds. On the other hand, there exist some natural ways (see Section 6.3) to construct closed 3 -manifolds from each homology cylinder. Therefore, by using its monoid structure, $\mathcal{C}_{g, 1}$ serves as a tool for classifying closed 3 -manifolds.

The aim of this paper is to study the structure of $\mathcal{C}_{g, 1}$ from an algebraic point of view. The main tools for that are invariants using noncommutative rings arising from 
group rings of fundamental groups of homology cylinders. The first half of this paper is occupied by defining the Magnus representation for homology cylinders and observing its fundamental properties. In the latter half, Cochran and Harvey's higherorder Alexander invariants are introduced and used to define numerical invariants for matrices obtained by the Magnus representation or associated Reidemeister torsions, whose entries are in noncommutative rings so that it is difficult to treat directly. This combination of the Magnus representation and higher-order Alexander invariants leads us to derive various facts about the structure of $\mathcal{C}_{g, 1}$ and to give applications to the theory of closed 3-manifolds.

The outline of this paper is as follows.

In Section 2, we first recall the definition of homology cylinders over $\Sigma_{g, 1}$ by following that of Garoufalidis-Levine [9] and Levine [21]. Then the monoid $\mathcal{C}_{g, 1}$ of homology cylinders and its quotient group $\mathcal{H}_{g, 1}$ by homology cobordisms are introduced. They are main objects of this paper. An example of homology cylinders comes from the mapping class group $\mathcal{M}_{g, 1}$ of $\Sigma_{g, 1}$. In fact, $\mathcal{M}_{g, 1}$ is contained in $\mathcal{C}_{g, 1}$ and $\mathcal{H}_{g, 1}$ as a subgroup. We use Stallings' theorem to give filtrations $\left\{\mathcal{C}_{g, 1}[k]\right\}_{k \geq 1}$ and $\left\{\mathcal{H}_{g, 1}[k]\right\}_{k \geq 1}$ of $\mathcal{C}_{g, 1}$ and $\mathcal{H}_{g, 1}$ respectively, which enable us to apply the Johnson-Morita theory used for $\mathcal{M}_{g, 1}$ (see Morita [24] for details) to homology cylinders. Note that, in Goussarov [10] and Habiro [11], the term "homology cylinders" indicates homology cylinders in $\mathcal{C}_{g, 1}[2]$.

In Section 3, which is the first main part of this paper, we study the Magnus representation

$$
r_{k}: \mathcal{C}_{g, 1} \longrightarrow G L\left(2 g, \mathcal{K}_{N_{k}}\right) \quad(k=2,3, \ldots)
$$

for homology cylinders, where $\mathcal{K}_{N_{k}}$ is a certain (skew) field. This "representation", which is in fact a crossed homomorphism, extends that for $\mathcal{M}_{g, 1}$ defined by Morita [24]. (See also Birman's book [2] for generalities of the ordinary Magnus representation as well as free differentials.) We can easily check that $r_{k}$ factors through $\mathcal{H}_{g, 1}$. One method for defining $r_{k}$ was already given in our article [28] as an analogue of the extension of the Gassner representation from pure braids to pure string links defined by Le Dimet [17]. More precisely, what we defined in [28] is a noncommutative generalization of the analogue of the Gassner representation. (In Section 4, the definition of a noncommutative generalization of the Gassner representation is described for completeness.) In this paper, we re-define the Magnus representation for $\mathcal{C}_{g, 1}$ and $\mathcal{H}_{g, 1}$ (Definition 3.3) by following Kirk-Livingston-Wang [16], where another and simplified way to define the Gassner representation for string links is given by using twisted (co)homology. Kirk-Livingston-Wang's method is also easier to give applications to the theory of 3-manifolds. After observing some fundamental properties of the Magnus representation, we see a method of the computation in Section 3.2. 
In Section 4, we apply the computational method given in Section 3.2 to homology cylinders obtained from pure string links by using Levine's construction in [21]. We will show that our Magnus representation contains the Gassner representation for string links. As a corollary, we see that $\mathcal{M}_{g, 1}$ is not a normal subgroup of $\mathcal{H}_{g, 1}$.

The latter half of this paper starts at Section 5 . We begin by a brief review of higherorder Alexander invariants due to Cochran [4] and Harvey [12; 13]. Higher-order Alexander invariants are invariants for finitely presentable groups interpreted as degrees of "noncommutative Alexander polynomials", which have some unclear ambiguity except their degrees. Historically, they were first defined for knot groups by Cochran, and then generalized for arbitrary finitely presentable groups by Harvey. Using them, Cochran and Harvey obtained various sharper results than those brought by the ordinary Alexander invariants: lower bounds on the knot genus or the Thurston norm, necessary conditions for realizing a given group as the fundamental group of some compact oriented 3-manifold, and so on. When we apply the theory of higher-order Alexander invariants to our situation, we shall need a slight generalization called torsion-degree functions because of the difference of localizations of noncommutative rings used in the Magnus representation and higher-order Alexander invariants (see Remark 3.2). Torsion-degree functions, whose definition occupies the middle of this section, are integer-valued maps

$$
d_{\Gamma}^{\psi}: M\left(\mathcal{K}_{\Gamma}\right) \longrightarrow \mathbb{Z} \quad\left(\psi \in H^{1}(\Gamma), \text { primitive }\right)
$$

from (not necessarily invertible) matrices $M\left(\mathcal{K}_{\Gamma}\right)$ with entries in the right field of fractions $\mathcal{K}_{\Gamma}=\mathbb{Z} \Gamma(\mathbb{Z} \Gamma-\{0\})^{-1}$ of the group ring of a group $\Gamma$ in a certain class. The construction of them is given by using the Dieudonné determinant and it is somewhat technical. However, the properties we need are summarized in Example 5.7, where we see that torsion-degree functions give a purely algebraic description of the machinery of higher-order Alexander invariants.

In Section 6, which is the second main part, we give various applications of torsiondegree functions to homology cylinders. We first apply them to the Magnus representation (Section 6.1) and associated Reidemeister torsions (Section 6.2). In some special cases, the results of these subsections are related to higher-order Alexander invariants of closed 3-manifolds associated with homology cylinders by deriving some factorization formulas (Theorems 6.8, 6.11 and 6.12) in Section 6.3. In Section 6.4, we observe the result of Section 6.2 more closely and show that there exist many families of torsion-degree functions which are homomorphisms on $\mathcal{C}_{g, 1}$ [2] and linearly independent of each other (Theorem 6.15 and Corollary 6.16) by using an argument similar to Harvey's Realization Theorem [12, Theorem 11.2]. From this, we immediately see that $\mathcal{C}_{g, 1}[2], \mathcal{C}_{g, 1}[3], \ldots$ are not finitely generated. 
In Section 7, we apply our machinery of torsion-degree functions to the automorphism group Aut $F_{n}^{\text {acy }}$ of the acyclic closure of a free group, which can be regarded as an enlargement of the automorphisms group of a free group. We show that the abelianization of certain subgroup of Aut $F_{n}^{\text {acy }}$ has infinite rank by using the linearly independence of torsion-degree functions.

\section{Homology cylinders}

Throughout the paper, we work in PL or smooth category. Let $\Sigma_{g, 1}$ be a compact connected oriented surface of genus $g \geq 1$ with one boundary component. We take a base point $p$ on the boundary of $\Sigma_{g, 1}$, and take $2 g$ loops $\gamma_{1}, \ldots, \gamma_{2 g}$ of $\Sigma_{g, 1}$ as shown in Figure 1. We consider them to be an embedded bouquet $R_{2 g}$ of $2 g$ circles tied at the base point $p \in \partial \Sigma_{g, 1}$. Then $R_{2 g}$ and the boundary loop $\zeta$ of $\Sigma_{g, 1}$ together with one 2-cell make up a standard cell decomposition of $\Sigma_{g, 1}$. The fundamental group $\pi_{1} \Sigma_{g, 1}$ of $\Sigma_{g, 1}$ is isomorphic to the free group $F_{2 g}$ of rank $2 g$ generated by $\gamma_{1}, \ldots, \gamma_{2 g}$, in which $\zeta=\prod_{i=1}^{g}\left[\gamma_{i}, \gamma_{g+i}\right]$.

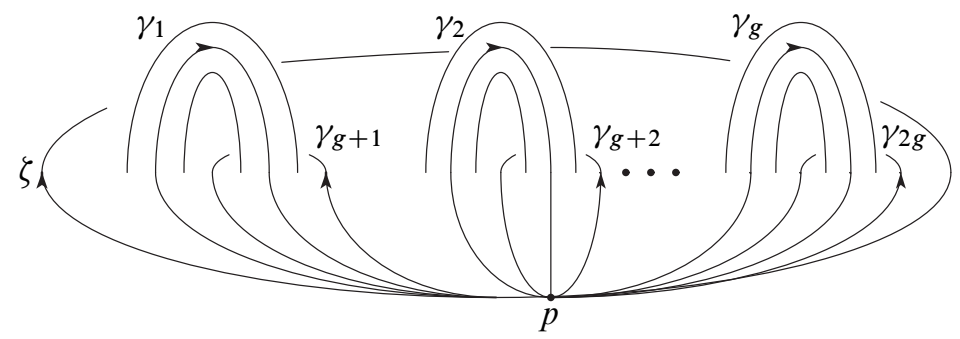

Figure 1: A standard cell decomposition of $\Sigma_{g, 1}$

A homology cylinder $\left(M, i_{+}, i_{-}\right)$(over $\left.\Sigma_{g, 1}\right)$, which has its origin in Goussarov [10], Habiro [11], Garoufalidis-Levine [9] and Levine [21], consists of a compact oriented 3-manifold $M$ and two embeddings $i_{+}, i_{-}: \Sigma_{g, 1} \rightarrow \partial M$ satisfying the following:

(1) $i_{+}$is orientation-preserving and $i_{-}$is orientation-reversing,

(2) $\partial M=i_{+}\left(\Sigma_{g, 1}\right) \cup i_{-}\left(\Sigma_{g, 1}\right)$ and $i_{+}\left(\Sigma_{g, 1}\right) \cap i_{-}\left(\Sigma_{g, 1}\right)=i_{+}\left(\partial \Sigma_{g, 1}\right)=i_{-}\left(\partial \Sigma_{g, 1}\right)$,

(3) $\left.i_{+}\right|_{\partial \Sigma_{g, 1}}=\left.i_{-}\right|_{\partial \Sigma_{g, 1}}$,

(4) $i_{+}, i_{-}: H_{*}\left(\Sigma_{g, 1}\right) \rightarrow H_{*}(M)$ are isomorphisms.

We denote $i_{+}(p)=i_{-}(p)$ by $p \in \partial M$ again and consider it to be the base point of $M$. We write $\left(M, i_{+}, i_{-}\right)$or simply $M$ for a homology cylinder. 
Two homology cylinders are said to be isomorphic if there exists an orientationpreserving diffeomorphism between the underlying 3-manifolds which is compatible with the embeddings of $\Sigma_{g, 1}$. We denote the set of isomorphism classes of homology cylinders by $\mathcal{C}_{g, 1}$. Given two homology cylinders $M=\left(M, i_{+}, i_{-}\right)$and $N=\left(N, j_{+}, j_{-}\right)$, we can construct a new homology cylinder $M \cdot N$ by

$$
M \cdot N=\left(M \cup_{i_{-} \circ\left(j_{+}\right)^{-1}} N, i_{+}, j_{-}\right) .
$$

Then $\mathcal{C}_{g, 1}$ becomes a monoid with the unit $1_{\mathcal{C}_{g, 1}}:=\left(\Sigma_{g, 1} \times I\right.$, id $\times 1$, id $\left.\times 0\right)$.

From the monoid $\mathcal{C}_{g, 1}$, we can construct the homology cobordism group $\mathcal{H}_{g, 1}$ of homology cylinders as in the following way. Two homology cylinders $M=\left(M, i_{+}, i_{-}\right)$ and $N=\left(N, j_{+}, j_{-}\right)$are homology cobordant if there exists a compact oriented 4manifold $W$ such that

(1) $\partial W=M \cup(-N) /\left(i_{+}(x)=j_{+}(x), i_{-}(x)=j_{-}(x)\right) \quad x \in \Sigma_{g, 1}$,

(2) the inclusions $M \hookrightarrow W, N \hookrightarrow W$ induce isomorphisms on the homology,

where $-N$ is $N$ with opposite orientation. We denote by $\mathcal{H}_{g, 1}$ the quotient set of $\mathcal{C}_{g, 1}$ with respect to the equivalence relation of homology cobordism. The monoid structure of $\mathcal{C}_{g, 1}$ induces a group structure of $\mathcal{H}_{g, 1}$. In the group $\mathcal{H}_{g, 1}$, the inverse of $\left(M, i_{+}, i_{-}\right)$is given by $\left(-M, i_{-}, i_{+}\right)$.

Example 2.1 For each element $\varphi$ of the mapping class group $\mathcal{M}_{g, 1}$ of $\Sigma_{g, 1}$, we can construct a homology cylinder $M_{\varphi} \in \mathcal{C}_{g, 1}$ by setting

$$
M_{\varphi}:=\left(\Sigma_{g, 1} \times I, \mathrm{id} \times 1, \varphi \times 0\right),
$$

where collars of $i_{+}\left(\Sigma_{g, 1}\right)$ and $i_{-}\left(\Sigma_{g, 1}\right)$ are stretched half-way along $\partial \Sigma_{g, 1} \times I$. As mentioned in Garoufalidis-Levine [9, Section 2.4] and Levine [21, Section 2.1], the existence of the homomorphisms $\sigma_{k}(k \geq 2)$ below shows that this construction gives injective homomorphisms $\mathcal{M}_{g, 1} \hookrightarrow \mathcal{C}_{g, 1}$ and $\mathcal{M}_{g, 1} \hookrightarrow \mathcal{H}_{g, 1}$ since $\bigcap_{k \geq 2} \operatorname{Ker}\left(\left.\sigma_{k}\right|_{\mathcal{M}_{g, 1}}\right)=$ $\{1\}$. From this, we can regard $\mathcal{C}_{g, 1}$ and $\mathcal{H}_{g, 1}$ as enlargements of $\mathcal{M}_{g, 1}$.

Let $N_{k}(G):=G /\left(\Gamma^{k} G\right)$ be the $k$-th nilpotent quotient of a group $G$, where we define $\Gamma^{1} G=G$ and $\Gamma^{l} G=\left[\Gamma^{l-1} G, G\right]$ for $l \geq 2$. For simplicity, we write $N_{k}(X)$ for $N_{k}\left(\pi_{1} X\right)$ where $X$ is a connected topological space, and write $N_{k}$ for $N_{k}\left(F_{2 g}\right)=$ $N_{k}\left(\Sigma_{g, 1}\right)$.

Let $\left(M, i_{+}, i_{-}\right)$be a homology cylinder. By definition, $i_{+}, i_{-}: \pi_{1} \Sigma_{g, 1} \rightarrow \pi_{1} M$ are both 2-connected, namely they induce isomorphisms on the first homology groups and epimorphisms on the second homology groups. Then, by Stallings' theorem [30, 
Theorem 3.4], $i_{+}, i_{-}: N_{k} \cong N_{k}(M)$ are isomorphisms for each $k \geq 2$. Using them, we obtain a monoid homomorphism

$$
\sigma_{k}: \mathcal{C}_{g, 1} \longrightarrow \text { Aut } N_{k} \quad\left(\left(M, i_{+}, i_{-}\right) \mapsto\left(i_{+}\right)^{-1} \circ i_{-}\right) .
$$

It can be easily checked that $\sigma_{k}$ induces a group homomorphism $\sigma_{k}: \mathcal{H}_{g, 1} \rightarrow$ Aut $N_{k}$. We define filtrations of $\mathcal{C}_{g, 1}$ and $\mathcal{H}_{g, 1}$ by

$$
\begin{aligned}
& \mathcal{C}_{g, 1}[1]:=\mathcal{C}_{g, 1}, \quad \mathcal{C}_{g, 1}[k]:=\operatorname{Ker}\left(\mathcal{C}_{g, 1} \stackrel{\sigma_{k}}{\longrightarrow} \text { Aut } N_{k}\right) \text { for } k \geq 2, \\
& \mathcal{H}_{g, 1}[1]:=\mathcal{H}_{g, 1}, \mathcal{H}_{g, 1}[k]:=\operatorname{Ker}\left(\mathcal{H}_{g, 1} \stackrel{\sigma_{k}}{\longrightarrow} \text { Aut } N_{k}\right) \text { for } k \geq 2 .
\end{aligned}
$$

(We follow the notation of [28]. Note that $\mathcal{H}_{g, 1}[k]$ is denoted by $\mathcal{F}_{k-1}^{w}\left(\mathcal{H}_{g}\right)$ in Levine [21].)

\section{The Magnus representation for homology cylinders}

We first summarize our notation. For a matrix $A$ with entries in a ring $R$, and a ring homomorphism $\varphi: R \rightarrow R^{\prime}$, we denote by ${ }^{\varphi} A$ the matrix obtained from $A$ by applying $\varphi$ to each entry. $A^{T}$ denotes the transpose of $A$. When $R=\mathbb{Z} G$ for a group $G$ or its right field of fractions (if exists), we denote by $\bar{A}$ the matrix obtained from $A$ by applying the involution induced from $\left(x \mapsto x^{-1}, x \in G\right)$ to each entry.

For a module $M$, we write $M^{n}$ and $M_{n}$ for the modules of column and row vectors with $n$ entries respectively.

For a connected CW-complex $X$ and its regular covering $X_{\Gamma}$ with respect to a homomorphism $\pi_{1} X \rightarrow \Gamma$, we consider the right action of $\Gamma$ on $X_{\Gamma}$ as in Harvey [12, Section 5]. Therefore the cellular chain complex $C_{*}\left(X_{\Gamma}\right)$ of $X_{\Gamma}$ becomes a right $\mathbb{Z} \Gamma$-module. For each $(\mathbb{Z} \Gamma, R)$-bimodule $A$, the twisted chain complex $C_{*}(X ; A)$ is given by the tensor product of the right $\mathbb{Z} \Gamma$-module $C_{*}\left(X_{\Gamma}\right)$ and the left $\mathbb{Z} \Gamma$-module $A$, so that $C_{*}(X ; A)$ and $H_{*}(X ; A)$ are right $R$-modules.

\subsection{Definition of the Magnus representation for homology cylinders}

In what follows, we fix an integer $k \geq 2$, which corresponds to the class of the nilpotent quotient. The following construction is based on Kirk-Livingston-Wang's work of the Gassner representation for string links in [16].

Let $\left(M, i_{+}, i_{-}\right) \in \mathcal{C}_{g, 1}$. By Stallings' theorem, $N_{k}$ and $N_{k}(M)$ are isomorphic. Since $N_{k}$ is a finitely generated torsion-free nilpotent group for each $k \geq 2$, we can embed $\mathbb{Z} N_{k}$ into the right field of fractions $\mathcal{K}_{N_{k}}:=\mathbb{Z} N_{k}\left(\mathbb{Z} N_{k}-\{0\}\right)^{-1}$. (See 
Section 5.) Similarly, we have $\mathbb{Z} N_{k}(M) \hookrightarrow \mathcal{K}_{N_{k}(M)}:=\mathbb{Z} N_{k}(M)\left(\mathbb{Z} N_{k}(M)-\{0\}\right)^{-1}$. We consider the fields $\mathcal{K}_{N_{k}}$ and $\mathcal{K}_{N_{k}(M)}$ to be local coefficient systems on $\Sigma_{g, 1}$ and $M$ respectively.

Lemma 3.1 (Cochran-Orr-Teichner [5, Proposition 2.10])

$$
i_{ \pm}: H_{*}\left(\Sigma_{g, 1}, p ; i_{ \pm}^{*} \mathcal{K}_{N_{k}(M)}\right) \rightarrow H_{*}\left(M, p ; \mathcal{K}_{N_{k}(M)}\right)
$$

are isomorphisms as right $\mathcal{K}_{N_{k}(M)}$-vector spaces.

Remark 3.2 A statement similar to Lemma 3.1 is true for the homology with coefficients in any left $\mathbb{Z} \pi_{1} M$-algebra $A$ satisfying the following property: Every matrix with entries in $\mathbb{Z} \pi_{1} M$ sent to an invertible one by the augmentation map $\mathbb{Z} \pi_{1} M \rightarrow \mathbb{Z}$ is also invertible in $A$. Note that $\mathcal{K}_{N_{k}(M)}$ satisfies this property (although $\mathcal{K}_{N_{k}(M)^{\psi}\left[t^{ \pm 1}\right]}$ in Section 5 does not in general).

Since the bouquet $R_{2 g}$ is a deformation retract of $\Sigma_{g, 1}$ relative to $p$, we have isomorphisms $\iota_{ \pm}: \mathcal{K}_{N_{k}(M)}^{2 g} \cong H_{1}\left(\Sigma_{g, 1}, p ; i_{ \pm}^{*} \mathcal{K}_{N_{k}(M)}\right)$ of right $\mathcal{K}_{N_{k}(M)}$-vector spaces defined as the inverse map of the composite of

$$
\begin{aligned}
H_{1}\left(\Sigma_{g, 1}, p ; i_{ \pm}^{*} \mathcal{K}_{N_{k}(M)} \cong H_{1}\left(R_{2 g}, p ; i_{ \pm}^{*} \mathcal{K}_{N_{k}(M)}\right)\right. & =C_{1}\left(R_{2 g}, p ; i_{ \pm}^{*} \mathcal{K}_{N_{k}(M)}\right) \\
& =C_{1}\left(\widetilde{R_{2 g}}\right) \otimes_{\pi_{1} R_{2 g}} i_{ \pm}^{*} \mathcal{K}_{N_{k}(M)} \\
& \cong \mathcal{K}_{N_{k}(M)}^{2 g}
\end{aligned}
$$

under a basis

$$
\left\{\widetilde{\gamma_{1}} \otimes 1, \ldots, \widetilde{\gamma_{2 g}} \otimes 1\right\} \subset C_{1}\left(\widetilde{R_{2 g}}\right) \otimes_{\pi_{1} R_{2 g}} i_{ \pm}^{*} \mathcal{K}_{N_{k}(M)},
$$

where $\widetilde{\gamma_{i}}$ is a lift of $\gamma_{i}$ on the universal covering $\widetilde{R_{2 g} g}$.

Definition 3.3 (1) For each $M=\left(M, i_{+}, i_{-}\right) \in \mathcal{C}_{g, 1}$, we denote by $r_{k}^{\prime}(M) \in$ $G L\left(2 g, \mathcal{K}_{N_{k}(M)}\right)$ the representation matrix of the right $\mathcal{K}_{N_{k}(M)}$-isomorphism:

$$
\begin{aligned}
\mathcal{K}_{N_{k}(M)}^{2 g} \underset{\iota_{-}}{\cong} H_{1}\left(\Sigma_{g, 1}, p ; i_{-}^{*} \mathcal{K}_{N_{k}(M)}\right) & \stackrel{\cong}{\underset{i_{-}}{\longrightarrow}} H_{1}\left(M, p ; \mathcal{K}_{N_{k}(M)}\right) \\
& \stackrel{\cong}{\stackrel{\cong}{i_{+}^{-1}}} H_{1}\left(\Sigma_{g, 1}, p ; i_{+}^{*} \mathcal{K}_{N_{k}(M)} \underset{\iota_{+}^{-1}}{\stackrel{\cong}{\longrightarrow}} \mathcal{K}_{N_{k}(M)}^{2 g}\right.
\end{aligned}
$$

(2) The Magnus representation for $\mathcal{C}_{g, 1}$ is the map $r_{k}: \mathcal{C}_{g, 1} \rightarrow G L\left(2 g, \mathcal{K}_{N_{k}}\right)$ which assigns to $M=\left(M, i_{+}, i_{-}\right) \in \mathcal{C}_{g, 1}$ the matrix ${ }_{+}^{-1} r_{k}^{\prime}(M)$. We call $r_{k}(M)$ the Magnus matrix of $M$. 
Remark 3.4 Since the base points of $\Sigma_{g, 1}, R_{2 g}$ and $M$ are the same, $r_{k}^{\prime}(M)$ and $r_{k}(M)$ depend only on the choice of a cell decomposition of $\Sigma_{g, 1}$ (or a basis of $\left.\pi_{1} \Sigma_{g, 1}\right)$. See also Proposition 3.10.

While we call $r_{k}(M)$ the Magnus "representation", it is actually a crossed homomorphism, namely we have the following.

Theorem 3.5 For $M_{1}, M_{2} \in \mathcal{C}_{g, 1}$, we have

$$
r_{k}\left(M_{1} \cdot M_{2}\right)=r_{k}\left(M_{1}\right) \cdot{ }^{\sigma_{k}\left(M_{1}\right)} r_{k}\left(M_{2}\right)
$$

Proof We write $M=M_{1} \cdot M_{2}$ for simplicity. Let $i: M_{1} \rightarrow M$ and $j: M_{2} \rightarrow M$ be the natural inclusions. Since $M=\left(M, i \circ i_{+}, j \circ j_{-}\right)$and $i \circ i_{-}=j \circ j_{+}$, the map

$$
\begin{aligned}
& H_{1}\left(\Sigma_{g, 1}, p ; j_{-}^{*} j^{*} \mathcal{K}_{N_{k}(M)}\right) \stackrel{j \circ j_{-}}{\longrightarrow} H_{1}\left(M, p ; \mathcal{K}_{N_{k}(M)}\right) \\
& \stackrel{\left(i \circ i_{+}\right)^{-1}}{\longrightarrow} H_{1}\left(\Sigma_{g, 1}, p ; i_{+}^{*} i^{*} \mathcal{K}_{N_{k}(M)}\right)
\end{aligned}
$$

is given as the composite of

$$
\begin{aligned}
H_{1}\left(\Sigma_{g, 1}, p ; j_{-}^{*} j^{*} \mathcal{K}_{N_{k}(M)}\right) \stackrel{j_{-}}{\longrightarrow} H_{1}\left(M_{2}, p ; j^{*} \mathcal{K}_{N_{k}(M)}\right) & \\
\qquad & \stackrel{j_{+}^{-1}}{\longrightarrow} H_{1}\left(\Sigma_{g, 1}, p ; j_{+}^{*} j^{*} \mathcal{K}_{N_{k}(M)}\right)
\end{aligned}
$$

and $\quad H_{1}\left(\Sigma_{g, 1}, p ; i_{-}^{*} i^{*} \mathcal{K}_{N_{k}(M)}\right) \stackrel{i_{-}}{\longrightarrow} H_{1}\left(M_{1}, p ; i^{*} \mathcal{K}_{N_{k}(M)}\right)$

$$
\stackrel{i_{+}^{-1}}{\longrightarrow} H_{1}\left(\Sigma_{g, 1}, p ; i_{+}^{*} i^{*} \mathcal{K}_{N_{k}(M)}\right)
$$

Hence

$$
\begin{aligned}
& r_{k}^{\prime}(M)={ }^{i} r_{k}^{\prime}\left(M_{1}\right) \cdot{ }^{j} r_{k}^{\prime}\left(M_{2}\right) \\
& \Longrightarrow{ }^{\left(i i_{+}\right)^{-1}} r_{k}^{\prime}(M)={ }^{\left(i i_{+}\right)^{-1} i} r_{k}^{\prime}\left(M_{1}\right) \cdot{ }^{\left(i i_{+}\right)^{-1} j} r_{k}^{\prime}\left(M_{2}\right) \\
& ={ }^{i_{+}^{-1}} r_{k}^{\prime}\left(M_{1}\right) \cdot{ }^{i_{+}^{-1}} i^{-1} j r_{k}^{\prime}\left(M_{2}\right) \\
& ={ }^{i_{+}^{-1}} r_{k}^{\prime}\left(M_{1}\right) \cdot{ }^{i_{+}^{-1} i_{-} j_{+}^{-1}} r_{k}^{\prime}\left(M_{2}\right) \\
& \Longrightarrow \quad r_{k}(M)=r_{k}\left(M_{1}\right) \cdot{ }^{\sigma_{k}\left(M_{1}\right)} r_{k}\left(M_{2}\right) \text {. }
\end{aligned}
$$

This completes the proof. 
Theorem 3.6 $r_{k}: \mathcal{C}_{g, 1} \rightarrow G L\left(2 g, \mathcal{K}_{N_{k}}\right)$ factors through $\mathcal{H}_{g, 1}$.

Proof Suppose $M_{1}=\left(M_{1}, i_{+}, i_{-}\right)$and $M_{2}=\left(M_{2}, j_{+}, j_{-}\right) \in \mathcal{C}_{g, 1}$ are homology cobordant by a homology cobordism $W$. Let $i: M_{1} \rightarrow W, j: M_{2} \rightarrow W$ be the natural inclusions. We may assume that $M_{1} \cup M_{2}$ is a subcomplex of $W$ and that $W$ has only one 0 -cell $p$. Since $\mathbb{Z} N_{k} \cong \mathbb{Z} N_{k}(W)$ by Stallings' theorem, we have $\mathcal{K}_{N_{k}(W)}:=\mathbb{Z} N_{k}(W)\left(\mathbb{Z} N_{k}(W)-\{0\}\right)^{-1}$. We write $I_{+}:=i \circ i_{+}=j \circ j_{+}$and $I_{-}:=i \circ i_{-}=j \circ j_{-}$. Then we have the following commutative diagram:

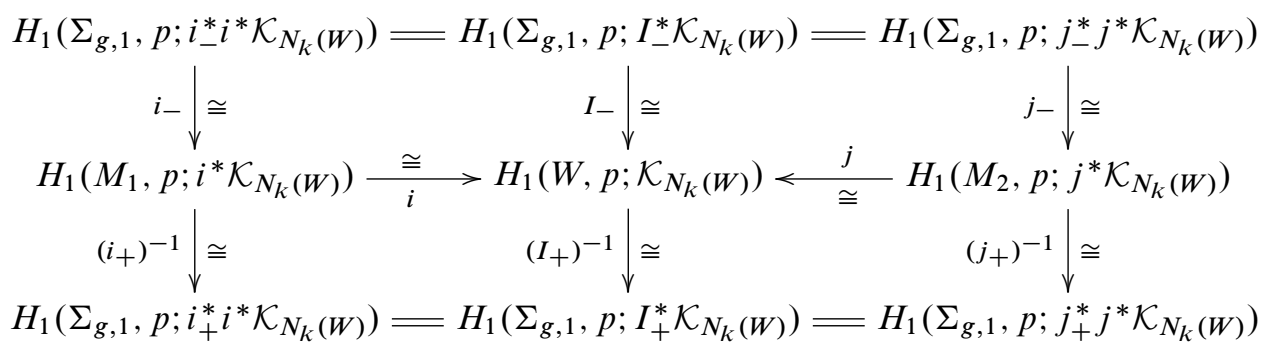

The left vertical maps give ${ }^{i} r_{k}^{\prime}\left(M_{1}\right)$ and the right ones give ${ }^{j} r_{k}^{\prime}\left(M_{2}\right)$. Applying $I_{+}^{-1}$, we obtain $r_{k}\left(M_{1}\right)=r_{k}\left(M_{2}\right)$.

Consequently, we obtain the Magnus representation $r_{k}: \mathcal{H}_{g, 1} \rightarrow G L\left(2 g, \mathcal{K}_{N_{k}}\right)$, which is a crossed homomorphism. If we restrict $r_{k}$ to $\mathcal{C}_{g, 1}[k]$ (resp. $\mathcal{H}_{g, 1}[k]$ ), it becomes a monoid (resp. group) homomorphism.

Example 3.7 For $\varphi \in \mathcal{M}_{g, 1} \hookrightarrow$ Aut $F_{2 g}$, we can obtain

$$
r_{k}\left(M_{\varphi}\right)=\rho^{\rho_{k}} \overline{\left(\frac{\partial \varphi\left(\gamma_{j}\right)}{\partial \gamma_{i}}\right)_{1 \leq i, j \leq 2 g}}
$$

where $\rho_{k}: \mathbb{Z} F_{2 g} \rightarrow \mathbb{Z} N_{k} \subset \mathcal{K}_{N_{k}}$ is the natural map and $\partial / \partial \gamma_{i}$ are free differentials. From this, we see that $r_{k}$ generalizes the original Magnus representation for $\mathcal{M}_{g, 1}$ in Morita [24].

\subsection{Computation of the Magnus matrix}

In Kirk-Livingston-Wang [16], the Gassner matrix of a string link is computed from the Wirtinger presentation of the fundamental group of its exterior, which gives a finite presentation whose deficiency coincides with the number of strings. Recall that the deficiency of a finite presentation $\left\{x_{1}, \ldots, x_{n} \mid r_{1}, \ldots, r_{m}\right\}$ of a finitely presentable group $G$ is $n-m$, and the deficiency of $G$ is the maximum of all over the deficiencies of finite presentations of $G$. In our context, we do not have such a useful method in general. 
Definition 3.8 For $\left(M, i_{+}, i_{-}\right) \in \mathcal{C}_{g, 1}$, a presentation of $\pi_{1} M$ is said to be admissible if it is of the form

$$
\left\langle i_{-}\left(\gamma_{1}\right), \ldots, i_{-}\left(\gamma_{2 g}\right), z_{1}, \ldots, z_{l}, i_{+}\left(\gamma_{1}\right), \ldots, i_{+}\left(\gamma_{2 g}\right) \mid r_{1}, \ldots, r_{2 g+l}\right\rangle .
$$

That is, it is a finite presentation with deficiency $2 g$ whose generating set contains $i_{-}\left(\gamma_{1}\right), \ldots, i_{-}\left(\gamma_{2 g}\right), i_{+}\left(\gamma_{1}\right), \ldots, i_{+}\left(\gamma_{2 g}\right)$ and is ordered as above.

Note that there does exist an admissible presentation of $\pi_{1} M$ for each $\left(M, i_{+}, i_{-}\right) \in$ $\mathcal{C}_{g, 1}$. Indeed, take a relative Morse function with no critical points of indices 0 and 3. Then $M$ can be seen as $\Sigma_{g, 1} \times I$ with some 1- and 2-handles. Since the Euler characteristics of $\Sigma_{g, 1} \times I$ and $M$ are the same, the numbers of the attached 1- and 2handles are the same. Therefore any presentation of $\pi_{1} M$ obtained from a presentation of $\pi_{1}\left(\Sigma_{g, 1} \times I\right)=F_{2 g}$ with deficiency $2 g$ by adding new generators and relations corresponding to the $1-$ and 2-handles has deficiency $2 g$ again. By adding $i_{ \pm}\left(\gamma_{j}\right)$ 's to the presentation with appropriate relations, we obtain an admissible presentation of $\pi_{1} M$. (See also Section 6.2.)

Now we see a method for computing Magnus matrices, which is based on KirkLivingston-Wang's one for computing Gassner matrices of string links [16, Section 4]. Comparing with the case of Kirk-Livingston-Wang, we need extra care about multiplications since we are dealing with more general situation by using noncommutative rings. However, almost the same argument holds.

Let $\left(M, i_{+}, i_{-}\right) \in \mathcal{C}_{g, 1}$. Given an admissible presentation of $\pi_{1} M$ as in Definition 3.8, we define $2 g \times(2 g+l), l \times(2 g+l)$ and $2 g \times(2 g+l)$ matrices $A, B, C$ by

$$
A=\overline{\left(\frac{\partial r_{j}}{\partial i_{-}\left(\gamma_{i}\right)}\right)} \underbrace{}_{\substack{1 \leq i \leq 2 g \\ 1 \leq j \leq 2 g+l}}, \quad B=\overline{\left(\frac{\partial r_{j}}{\partial z_{i}}\right)} \underbrace{}_{\substack{1 \leq i \leq l \\ 1 \leq j \leq 2 g+l}}, \quad C=\frac{\partial r_{j}}{\partial i_{+}\left(\gamma_{i}\right)})_{\substack{1 \leq i \leq 2 g \\ 1 \leq j \leq 2 g+l}}
$$

at $\mathbb{Z} N_{k}(M)$, namely we apply the natural map

$$
\mathbb{Z}\left\langle i_{-}\left(\gamma_{1}\right), \ldots, i_{-}\left(\gamma_{2 g}\right), z_{1}, \ldots, z_{l}, i_{+}\left(\gamma_{1}\right), \ldots, i_{+}\left(\gamma_{2 g}\right)\right\rangle \rightarrow \mathbb{Z} \pi_{1} M \rightarrow \mathbb{Z} N_{k}(M)
$$

to each entry of the matrices obtained by free differentials.

Proposition 3.9 As matrices with entries in $\mathcal{K}_{N_{k}(M)}$, we have the following.

(1) The square matrix $\left(\begin{array}{l}A \\ B\end{array}\right)$ is invertible.

(2) The following equality holds:

$$
r_{k}^{\prime}(M)=-C\left(\begin{array}{l}
A \\
B
\end{array}\right)^{-1}\left(\begin{array}{c}
I_{2 g} \\
0_{(l, 2 g)}
\end{array}\right) .
$$

Algebraic $8 \mathcal{G}$ Geometric Topology, Volume 8 (2008) 
In particular, the Magnus matrix $r_{k}^{\prime}(M)$ can be computed from any admissible presentation of $\pi_{1} M$.

Proof (1) Let $\mathfrak{t}: \mathbb{Z} N_{k}(M) \rightarrow \mathbb{Z}$ be the augmentation map. ${ }^{\mathfrak{t}}\left({ }_{B}^{A}\right)$ gives a presentation matrix of $H_{1}(M) / \Phi_{+}$, where $\Phi_{+}$is the subgroup of $H_{1}(M)$ generated by $i_{+}\left(\gamma_{1}\right), \ldots, i_{+}\left(\gamma_{2 g}\right)$. (See Fox [7] for this fact through the concept of presentations of a pair of groups.) By definition, $H_{1}(M) / \Phi_{+}=0$, and we have an exact sequence

$$
\mathbb{Z}^{2 g+l} \stackrel{\left.\mathfrak{t}^{(} \begin{array}{l}
A \\
B
\end{array}\right) .}{\longrightarrow} \mathbb{Z}^{2 g+l} \longrightarrow H_{1}(M) / \Phi_{+}=0 .
$$

By the Hopfian property of $\mathbb{Z}^{2 g+l}$, we see that ${ }^{\mathfrak{t}}\left({ }_{B}^{A}\right)$ is invertible. (1) follows from this. (See Remark 3.2.)

(2) By a standard argument using Eilenberg-MacLane spaces, we can assume that a given admissible presentation is obtained from a cell decomposition of $M$. We fix isomorphisms $\mathcal{K}_{N_{k}(M)}^{4 g+l} \cong C_{1}\left(M, p ; \mathcal{K}_{N_{k}(M)}\right)$ and $\mathcal{K}_{N_{k}(M)}^{2 g+l} \cong C_{2}\left(M, p ; \mathcal{K}_{N_{k}(M)}\right)$ by using the ordered bases $\left\langle i_{-}\left(\gamma_{1}\right), \ldots, i_{-}\left(\gamma_{2 g}\right), z_{1}, \ldots, z_{l}, i_{+}\left(\gamma_{1}\right), \ldots, i_{+}\left(\gamma_{2 g}\right)\right\rangle$ and $\left\langle r_{1}, \ldots, r_{2 g+l}\right\rangle$ respectively. The boundary map

$$
\text { д: } C_{2}\left(M, p ; \mathcal{K}_{N_{k}(M)}\right) \rightarrow C_{1}\left(M, p ; \mathcal{K}_{N_{k}(M)}\right)
$$

is given by multiplying

$$
\left(\begin{array}{l}
A \\
B \\
C
\end{array}\right)
$$

from the left. Let $e_{i}=\left(0_{(1, i-1)}, 1,0_{(1,2 g-i)}\right)^{T} \in \mathcal{K}_{N_{k}(M)}^{2 g}$, where we write $0_{(k, l)}$ for the zero matrix of size $k \times l$. By the definition of $r_{k}^{\prime}(M)$, two 1-cycles

$$
\left(\begin{array}{c}
e_{i} \\
0_{(l, 1)} \\
0_{(2 g, 1)}
\end{array}\right) \text { and } \quad\left(\begin{array}{c}
0_{(2 g, 1)} \\
0_{(l, 1)} \\
r_{k}^{\prime}(M) e_{i}
\end{array}\right)
$$

in $\mathcal{K}_{N_{k}(M)}^{4 g+l} \cong C_{1}\left(M, p ; \mathcal{K}_{N_{k}(M)}\right)$ are homologous, so that there exists $x_{i} \in \mathcal{K}_{N_{k}(M)}^{2 g+l} \cong$ $C_{2}\left(M, p ; \mathcal{K}_{N_{k}(M)}\right)$ satisfying

$$
\left(\begin{array}{c}
e_{i} \\
0_{(l, 1)} \\
0_{(2 g, 1)}
\end{array}\right)-\left(\begin{array}{c}
0_{(2 g, 1)} \\
0_{(l, 1)} \\
r_{k}^{\prime}(M) e_{i}
\end{array}\right)=\left(\begin{array}{l}
A \\
B \\
C
\end{array}\right) x_{i} \in C_{1}\left(M, p ; \mathcal{K}_{N_{k}(M)}\right)
$$

Algebraic 83 Geometric Topology, Volume 8 (2008) 
We put $X:=\left(x_{1}, \ldots, x_{2 g}\right) \in C_{2}\left(M, p ; \mathcal{K}_{N_{k}(M)}\right)_{2 g}$. Then we have

$$
\left(\begin{array}{c}
I_{2 g} \\
0_{(l, 2 g)} \\
0_{2 g}
\end{array}\right)-\left(\begin{array}{c}
0_{2 g} \\
0_{(l, 2 g)} \\
r_{k}^{\prime}(M)
\end{array}\right)=\left(\begin{array}{c}
A \\
B \\
C
\end{array}\right) X \in C_{1}\left(M, p ; \mathcal{K}_{N_{k}(M)}\right)_{2 g}
$$

where $0_{k}:=0_{(k, k)}$. This equality is split into

$$
\left(\begin{array}{c}
I_{2 g} \\
0_{(l, 2 g)}
\end{array}\right)=\left(\begin{array}{l}
A \\
B
\end{array}\right) X
$$

and $-r_{k}^{\prime}(M)=C X$. Eliminating $X$ by using (1), we obtain the equality of (2).

Next, we derive a formula for changing a basis of $\pi_{1} \Sigma_{g, 1}$. For $\left(M, i_{+}, i_{-}\right) \in \mathcal{C}_{g, 1}$, we take an admissible presentation of $\pi_{1} M$ as in Definition 3.8 and construct the matrices $A, B, C$ as before. Let $\gamma_{1}^{\prime}, \ldots, \gamma_{2 g}^{\prime}$ be another basis of $\pi_{1} \Sigma_{g, 1}$. We can take $\varphi \in$ Aut $\pi_{1} \Sigma_{g, 1}$ such that $\gamma_{i}^{\prime}=\varphi\left(\gamma_{i}\right)$ for $i=1, \ldots, 2 g$. Note that

$$
\left(\frac{\partial \varphi\left(\gamma_{i}\right)}{\partial \gamma_{j}}\right)_{1 \leq i, j \leq 2 g}
$$

is invertible as a matrix with entries in $\mathbb{Z} \pi_{1} \Sigma_{g, 1}$. In fact, we have

$$
\begin{aligned}
& \left(\frac{\partial \varphi\left(\gamma_{i}\right)}{\partial \gamma_{j}}\right)_{1 \leq i, j \leq 2 g}^{-1}={ }^{\varphi}\left(\frac{\partial \varphi^{-1}\left(\gamma_{i}\right)}{\partial \gamma_{j}}\right)_{1 \leq i, j \leq 2 g}, \\
& {\overline{\left(\frac{\partial \varphi\left(\gamma_{j}\right)}{\partial \gamma_{i}}\right)_{1 \leq i, j \leq 2 g}}}^{-1}={\overline{\left(\frac{\partial \varphi^{-1}\left(\gamma_{j}\right)}{\partial \gamma_{i}}\right)_{1 \leq i, j \leq 2 g}}} \text {, }
\end{aligned}
$$

which are deduced from the fundamental formula of free calculus (see Birman [2, Proposition 3.4]).

Proposition 3.10 Let $r_{k}^{\varphi}(M)$ be the Magnus matrix corresponding to the new basis. Then the following holds:

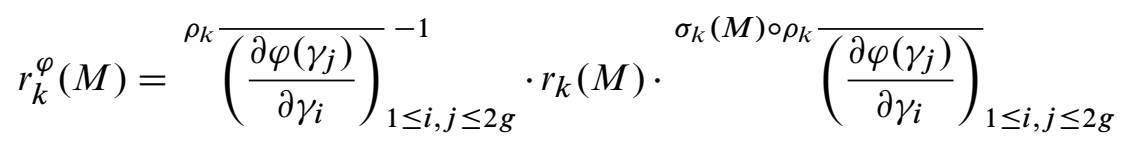

Algebraic 83 Geometric Topology, Volume 8 (2008) 
Proof We have the following admissible presentation of $\pi_{1} M$ with respect to $\gamma_{1}^{\prime}, \ldots$, $\gamma_{2 g}^{\prime}$ :

$$
\pi_{1} M \cong\left\langle\begin{array}{l|l}
i_{-}\left(\gamma_{1}^{\prime}\right), \ldots, i_{-}\left(\gamma_{2 g}^{\prime}\right) & \\
i_{-}\left(\gamma_{1}\right), \ldots, i_{-}\left(\gamma_{2 g}\right) & i_{-}\left(\gamma_{1}^{\prime}\right) i_{-}\left(\varphi\left(\gamma_{1}\right)\right)^{-1}, \ldots, i_{-}\left(\gamma_{2 g}^{\prime}\right) i_{-}\left(\varphi\left(\gamma_{2 g}\right)\right)^{-1} \\
z_{1}, \ldots, z_{l} & r_{1}, \ldots r_{2 g+l} \\
i_{+}\left(\gamma_{1}\right), \ldots, i_{+}\left(\gamma_{2 g}\right) & i_{+}\left(\gamma_{1}^{\prime}\right) i_{+}\left(\varphi\left(\gamma_{1}\right)\right)^{-1}, \ldots, i_{+}\left(\gamma_{2 g}^{\prime}\right) i_{+}\left(\varphi\left(\gamma_{2 g}\right)\right)^{-1}
\end{array}\right\rangle .
$$

The matrices $A^{\prime}, B^{\prime}, C^{\prime}$ corresponding to this presentation are given by

$$
\begin{aligned}
& A^{\prime}=\left(\begin{array}{lll}
I_{2 g} & 0_{(2 g, 2 g+l)} & 0_{2 g}
\end{array}\right), \\
& B^{\prime}=\left(\begin{array}{ccc}
\left.-i_{-} \circ \rho_{k} \frac{\partial \varphi\left(\gamma_{j}\right)}{\partial \gamma_{i}}\right)_{1 \leq i, j \leq 2 g} & A & 0_{2 g} \\
0_{(l, 2 g)} & B & 0_{(l, 2 g)} \\
0_{2 g} & & \left.C-\rho_{+} \circ \rho_{k} \frac{\partial \varphi\left(\gamma_{j}\right)}{\partial \gamma_{i}}\right)_{1 \leq i, j \leq 2 g}
\end{array}\right), \\
& C^{\prime}=\left(\begin{array}{lll}
0_{2 g} & \left.0_{(2 g, 2 g}+l\right) & I_{2 g}
\end{array}\right) .
\end{aligned}
$$

Put $J_{k}:=\rho_{k}{\overline{\left(\frac{\partial \varphi\left(\gamma_{j}\right)}{\partial \gamma_{i}}\right)}}_{1 \leq i, j \leq 2 g}$. Then

$$
\begin{aligned}
& \left(\begin{array}{c}
A^{\prime} \\
B^{\prime}
\end{array}\right)^{-1}=\left(\begin{array}{cc|c}
I_{2 g} & 0_{(2 g, 2 g+l)} & 0_{2 g} \\
-{ }^{i} J_{k} & A & 0_{2 g} \\
0_{(l, 2 g)} & B & 0_{(l, 2 g)} \\
\hline 0_{2 g} & C & -{ }^{i+} J_{k}
\end{array}\right)^{-1}=\left(\begin{array}{cc|c}
Z & 0_{(4 g+l, 2 g)} \\
i_{+} J_{k}^{-1}\left(0_{2 g}\right. & C) Z & -{ }^{i+} J_{k}^{-1}
\end{array}\right),
\end{aligned}
$$

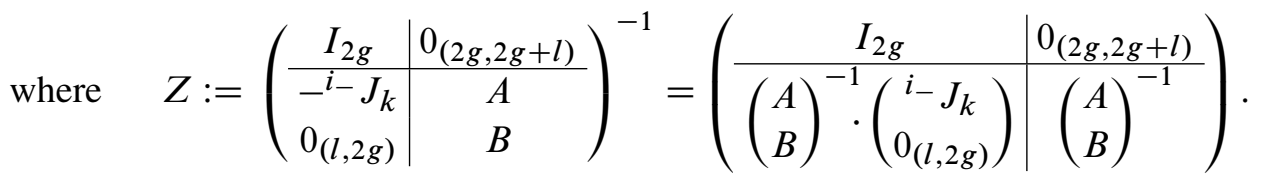

Using Proposition 3.9 (2), we have

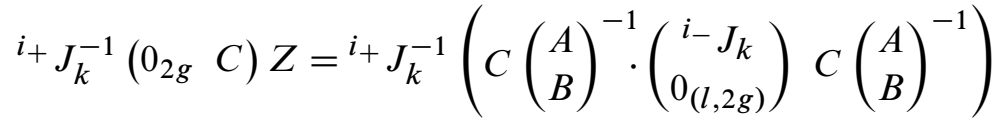

$$
\begin{aligned}
& =\left(-^{i+} J_{k}^{-1} r_{k}^{\prime}(M)^{i-} J_{k}{ }^{i}+J_{k}^{-1} C\left(\begin{array}{l}
A \\
B
\end{array}\right)^{-1}\right) \text {. }
\end{aligned}
$$


Therefore

$$
\begin{aligned}
& { }^{i}+r_{k}^{\varphi}(M)=-C^{\prime}\left(\begin{array}{l}
A^{\prime} \\
B^{\prime}
\end{array}\right)^{-1}\left(\begin{array}{c}
I_{2 g} \\
0_{(4 g+l, 2 g)}
\end{array}\right)
\end{aligned}
$$

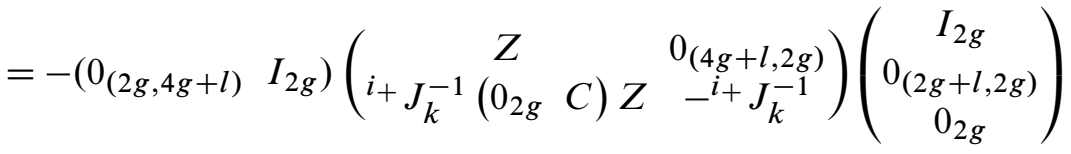

$$
\begin{aligned}
& =-{ }^{i_{+}} J_{k}^{-1}\left(\begin{array}{ll}
0_{2 g} & C
\end{array}\right) Z\left(\begin{array}{c}
I_{2 g} \\
0 \\
0_{(2 g+l, 2 g)}
\end{array}\right)={ }^{i_{+}} J_{k}^{-1} r_{k}^{\prime}(M)^{i_{-}} J_{k} .
\end{aligned}
$$

Applying $i_{+}^{-1}$ to each entry, we obtain the formula.

\section{Example: Relationship to the Gassner representation for string links}

In [21, Section 3], Levine gave a method for constructing homology cylinders from pure string links. By this, we can obtain many explicit examples of homology cylinders not belonging to the subgroup $\mathcal{M}_{g, 1}$. Here we consider Magnus matrices of such homology cylinders and see a relationship between the Gassner representation for string links and our representation.

First, we recall the definition of pure string links and describe a noncommutative generalization of the Gassner representation, whose original commutative version was defined by Le Dimet [17] and Kirk-Livingston-Wang [16]. Note that our convention differs from theirs.

Let $D^{2}$ be a 2 -dimensional disk. Given $n \geq 1$, we fix $n$ points $p_{1}, \ldots, p_{n}$ in the interior of $D^{2}$. An $n$-component pure string link is a smooth embedding of the disjoint union of ordered $n$ unit intervals into $D^{2} \times I$ sending 0 and 1 of $i$-th interval to $p_{i} \times\{0\}$ and $p_{i} \times\{1\}$ respectively. The set $\mathcal{L}_{n}$ of isotopy classes of $n$-component pure string links has a natural monoid structure. For $L \in \mathcal{L}_{n}$, its image in $D^{2} \times I$ is denoted by $L$ again. Let $D_{n}^{0}:=D^{2}-\left\{p_{1}, \ldots, p_{n}\right\} . \pi_{1} D_{n}^{0}$ is the free group generated by $n$ loops $\delta_{1}, \ldots, \delta_{n}$ as in Figure 2 .

We write $i_{0}$ and $i_{1}$ for the natural embeddings of $D_{n}^{0}$ into $D^{2} \times\{0\}$ and $D^{2} \times\{1\}$ respectively. It is easily checked that $i_{0}, i_{1}: \pi_{1} D_{n}^{0} \rightarrow \pi_{1}\left(D^{2} \times I-L\right)$ are 2-connected for each $L \in \mathcal{L}_{n}$. Hence they induce isomorphisms between $N_{k}^{0}:=N_{k}\left(\pi_{1} D_{n}^{0}\right)$ and $N_{k}(L):=N_{k}\left(\pi_{1}\left(D^{2} \times I-L\right)\right)$. 


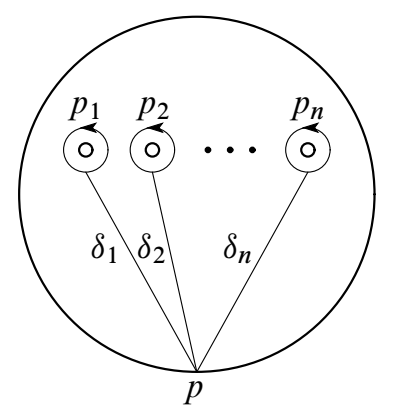

Figure 2: A standard basis of $\pi_{1} D_{n}^{0}$

Fix an integer $k \geq 2$. Cochran-Orr-Teichner's argument [5, Proposition 2.10] is used to show that $i_{j}: H_{1}\left(D_{n}^{0}, p ; i_{j}^{*} \mathcal{K}_{N_{k}(L)}\right) \rightarrow H_{1}\left(D^{2} \times I-L, p \times I ; \mathcal{K}_{N_{k}(L)}\right)$ are isomorphisms for $j=0,1$. The natural isomorphisms $\iota_{j}: \mathcal{K}_{N_{k}(L)}^{n} \cong H_{1}\left(D_{n}^{0}, p ; i_{j}^{*} \mathcal{K}_{N_{k}(L)}\right)$ are obtained by using the above basis of $\pi_{1} D_{n}^{0}$.

Definition 4.1 (1) For each $L \in \mathcal{L}_{n}$, we denote by $r_{G, k}^{\prime}(L) \in G L\left(n, \mathcal{K}_{N_{k}(L)}\right)$ the representation matrix of the right $\mathcal{K}_{N_{k}(L)}$-isomorphism

$$
\begin{aligned}
\mathcal{K}_{N_{k}(L)}^{n} \underset{\iota_{0}}{\cong} H_{1}\left(D_{n}^{0}, p ; i_{0}^{*} \mathcal{K}_{N_{k}(L)}\right) & \stackrel{\cong}{i_{0}} H_{1}\left(D^{2} \times I-L, p \times I ; \mathcal{K}_{N_{k}(L)}\right) \\
& \stackrel{\cong}{\underset{i_{1}^{-1}}{\longrightarrow}} H_{1}\left(D_{n}^{0}, p ; i_{1}^{*} \mathcal{K}_{N_{k}(L)}\right) \stackrel{\cong}{\stackrel{\iota_{1}^{-1}}{\longrightarrow}} \mathcal{K}_{N_{k}(L)}^{n} .
\end{aligned}
$$

(2) The Gassner representation for $\mathcal{L}_{n}$ is the map $r_{G, k}: \mathcal{L}_{n} \rightarrow G L\left(n, \mathcal{K}_{N_{k}^{0}}\right)$ which assigns to $L \in \mathcal{L}_{n}$ the matrix ${ }^{i}{ }_{1}^{-1} r_{G, k}^{\prime}(L)$. We call $r_{G, k}(L)$ the Gassner matrix of $L$.

$r_{G, k}$ is a crossed homomorphism in a suitable sense. Note that $r_{G, 2}$ corresponds to the original version.

As mentioned in Kirk-Livingston-Wang [16, Section 4], for $L \in \mathcal{L}_{n}$, the Wirtinger presentation gives a presentation of $\pi_{1}\left(D^{2} \times I-L\right)$ of the form

$$
\left\langle i_{0}\left(\delta_{1}\right), \ldots, i_{0}\left(\delta_{n}\right), z_{1}, \ldots, z_{l}, i_{1}\left(\delta_{1}\right), \ldots, i_{1}\left(\delta_{n}\right) \mid r_{1}, \ldots, r_{n+l}\right\rangle .
$$

Then the computation of $r_{G, k}(L)$ goes parallel to that of Magnus matrix in Section 3.2 and we omit it.

Next we recall Levine's construction [21, Section 3]. For a $g$-component pure string link $L \in \mathcal{L}_{g}$, we now construct a homology cylinder $M_{L} \in \mathcal{C}_{g, 1}$ as follows. Consider 


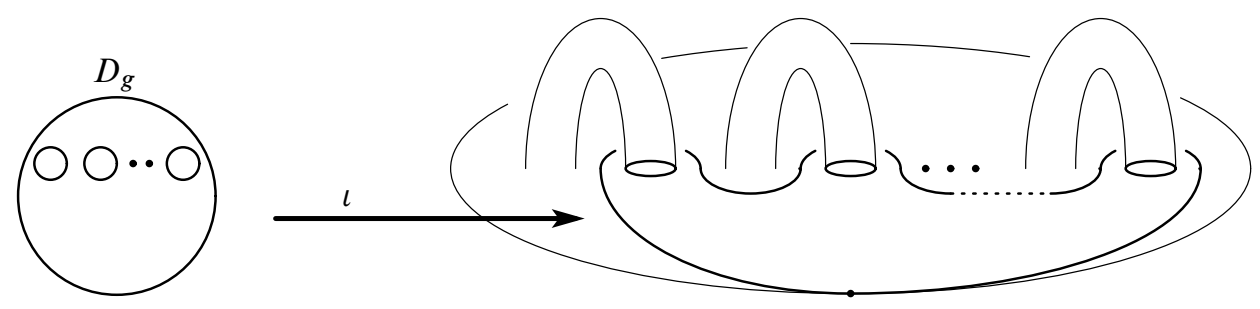

Figure 3

a closed tubular neighborhood of the loops $\gamma_{g+1}, \gamma_{g+2}, \ldots, \gamma_{2 g}$ in Figure 1 to be the image of an embedding $\iota: D_{g} \hookrightarrow \Sigma_{g, 1}$ of a $g$-holed disk $D_{g}$ as in Figure 3.

Let $C$ be the complement of an open tubular neighborhood of $L$ in $D^{2} \times I$. For each choice of a framing of $L$, a homeomorphism $h$ : $\partial C \cong \partial\left(\iota\left(D_{g}\right) \times I\right)$ is fixed. Then the manifold $M_{L}$ obtained from $\Sigma_{g, 1} \times I$ by removing $\iota\left(D_{g}\right) \times I$ and regluing $C$ by $h$ becomes a homology cylinder. Levine showed that this construction gives an injective monoid homomorphism $\mathcal{L}_{g} \rightarrow \mathcal{C}_{g, 1}$, and moreover, it induces an injective homomorphism $\mathcal{S}_{g} \rightarrow \mathcal{H}_{g, 1}$ from the concordance group $\mathcal{S}_{g}$ of pure string links to $\mathcal{H}_{g, 1}$. In particular, the (smooth) knot concordance group, which coincides with $\mathcal{S}_{1}$, is embedded in $\mathcal{H}_{g, 1}$. If we restrict these embeddings to the pure braid group, which is a subgroup of $\mathcal{L}_{g}$ and $\mathcal{S}_{g}$, their images are contained in $\mathcal{M}_{g, 1}$. We can easily check that $r_{G, k}$ factors through $\mathcal{S}_{g}$.

A relationship between the Gassner representation $r_{G, k}$ and the Magnus representation $r_{k}$ is given as follows.

Theorem 4.2 For any pure string link $L \in \mathcal{L}_{g}, r_{k}\left(M_{L}\right)=\left(\begin{array}{cc}* & 0 \\ * & r_{G, k}(L)\end{array}\right)$.

We mention two remarks about this theorem. First we identify $\pi_{1} D_{g} \cong \pi_{1} D_{g}^{0}=$ $\left\langle\delta_{1}, \ldots, \delta_{g}\right\rangle$ with the subgroup $\left\langle\gamma_{g+1}, \ldots, \gamma_{2 g}\right\rangle$ of $\pi_{1} \Sigma_{g, 1}$ by the correspondence $\left(\delta_{i} \mapsto \gamma_{g+i}\right)$ for $i=1, \ldots g$. Then the maps $\pi_{1} D_{g}^{0} \hookrightarrow \pi_{1} \Sigma_{g, 1} \rightarrow \pi_{1} D_{g}^{0}$, where the second map sends $\gamma_{1}, \ldots, \gamma_{g}$ to 1 , show that $N_{k}^{0} \subset N_{k}$ and $\mathcal{K}_{N_{k}^{0}} \subset \mathcal{K}_{N_{k}}$. Second, the embeddings $\mathcal{L}_{g} \hookrightarrow \mathcal{C}_{g, 1}$ and $\mathcal{S}_{g} \hookrightarrow \mathcal{H}_{g, 1}$ have ambiguity with respect to framings. However we can see that the lower right part of $r_{k}\left(M_{L}\right)$ is independent of the framings.

Proof of Theorem 4.2 To obtain a suitable admissible presentation of $\pi_{1} M_{L}$, we divide $M_{L}$ into two parts $X$ and $C$ as follows.

We take $g$ points $q_{1}, \ldots, q_{g}$ and $g$ paths $l_{j}$ from the base point $p$ to $q_{j}$ as in Figure 4 . 


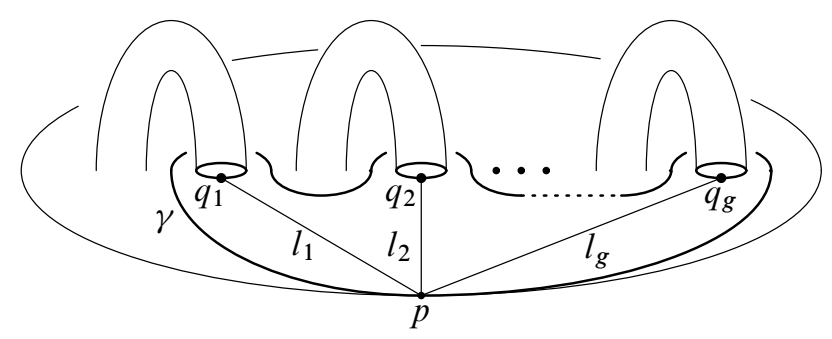

Figure 4

Let $X$ be the union of $\overline{\Sigma_{g, 1} \times I-\iota\left(D_{g}\right) \times I}$ and $2 g$ paths $i_{+}\left(l_{j}\right)$ and $i_{-}\left(l_{j}\right)(j=$ $1, \ldots, g)$. Then

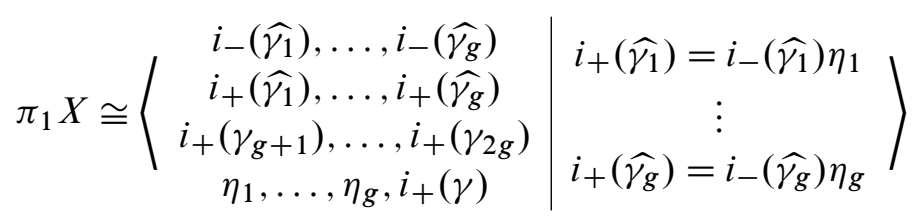

where the loop $\widehat{\gamma_{j}}:=\left[\gamma_{1}, \gamma_{g+1}\right] \cdots\left[\gamma_{j-1}, \gamma_{g+j-1}\right] \gamma_{j}$ satisfies $\widehat{\gamma_{j}} \cap \iota\left(D_{g}\right)=l_{j}, \gamma$ is the loop corresponding to the outer boundary of $\iota\left(D_{g}\right)$ and $\eta_{j}$ is the composite of paths $i_{-}\left(l_{j}\right), \overrightarrow{i_{-}\left(q_{j}\right) i_{+}\left(q_{j}\right)}$ and $i_{+}\left(l_{j}^{-1}\right)$. We denote by $C$ the complement of an open tubular neighborhood of $L$ in $D^{2} \times I$ as before.

$$
\pi_{1} C \cong\left\langle i_{-}\left(\gamma_{g+1}\right), \ldots, i_{-}\left(\gamma_{2 g}\right), z_{1}, \ldots, z_{l}, i_{+}\left(\gamma_{g+1}\right), \ldots, i_{+}\left(\gamma_{2 g}\right) \mid r_{1}, \ldots r_{g+l}\right\rangle
$$

is given by the Wirtinger presentation of $D^{2} \times I-L$. We glue $C$ to $X$ by using a fixed framing. Then it is easily seen that $\pi_{1}(X \cap C)$ is the free group generated by $\left\{i_{+}\left(\gamma_{g+1}\right), \ldots, i_{+}\left(\gamma_{2 g}\right), \eta_{1}, \ldots, \eta_{g}, i_{+}(\gamma)\right\}$.

Using the above decomposition, we obtain

$$
\pi_{1} M_{L} \cong\left(\begin{array}{c|c}
i_{-}\left(\widehat{\gamma_{1}}\right), \ldots, i_{-}\left(\widehat{\gamma_{g}}\right) & i_{+}\left(\widehat{\gamma_{g}}\right)=i_{-}\left(\widehat{\gamma_{1}}\right) \widehat{\eta_{1}} \\
z_{1}, \ldots, i_{-}\left(z_{2 g}\right) & \vdots \\
i_{+}\left(\widehat{\gamma_{1}}\right), \ldots, i_{+}\left(\widehat{\gamma_{g}}\right) & i_{+}\left(\widehat{\gamma_{g}}\right)=i_{-}\left(\widehat{\gamma_{g}}\right) \widehat{\eta_{g}}
\end{array}\right\rangle
$$

where each $\widehat{\eta_{i}}$ is a word in $i_{-}\left(\gamma_{g+1}\right), \ldots, i_{-}\left(\gamma_{2 g}\right), z_{1}, \ldots, z_{l}, i_{+}\left(\gamma_{g+1}\right), \ldots, i_{+}\left(\gamma_{2 g}\right)$ which depends on the framing. Rewrite the above presentation by using $i_{+}\left(\gamma_{j}\right)$ 's and $i_{-}\left(\gamma_{j}\right)$ 's instead of $i_{+}\left(\widehat{\gamma_{j}}\right)$ 's and $i_{-}\left(\widehat{\gamma_{j}}\right)$ 's. This process does not affect generators $i_{-}\left(\gamma_{g+j}\right), z_{j}, i_{+}\left(\gamma_{g+j}\right)$ and relations $r_{j}$. From the resulting admissible presentation, we can compute the Magnus matrix of $M_{L}$ as seen in Section 3.2. So our claim follows 
by comparing it with the method for calculating $r_{G, k}(L)$. (The most important point is that the admissible presentation of $\pi_{1} M_{L}$ obtained contains the Wirtinger presentation of $\pi_{1} C=\pi_{1}\left(D^{2} \times I-L\right)$.) It is straightforward and we omit the details.

Corollary 4.3 $\mathcal{M}_{g, 1}$ is not a normal subgroup of $\mathcal{H}_{g, 1}$ for $g \geq 3$.

Proof In [16, Section 7], Kirk-Livingston-Wang gave 3-component pure string links denoted by $L_{5}$ and $L_{6}$ having the condition that $L_{5}$ is a pure braid, while the conjugate $L_{6} L_{5} L_{6}^{-1} \in \mathcal{S}_{3}$ is not. To show that $L_{6} L_{5} L_{6}^{-1}$ is not a pure braid, they use the fact that $r_{G, 2}\left(L_{6} L_{5} L_{6}^{-1}\right)$ has an entry not belonging to $\mathbb{Z} N_{2}\left(D_{3}^{0}\right)$. Then our claim follows by applying Theorem 4.2 to this example.

Example 4.4 Let $L$ be a 2-component pure string link as depicted in the left of Figure 5 .
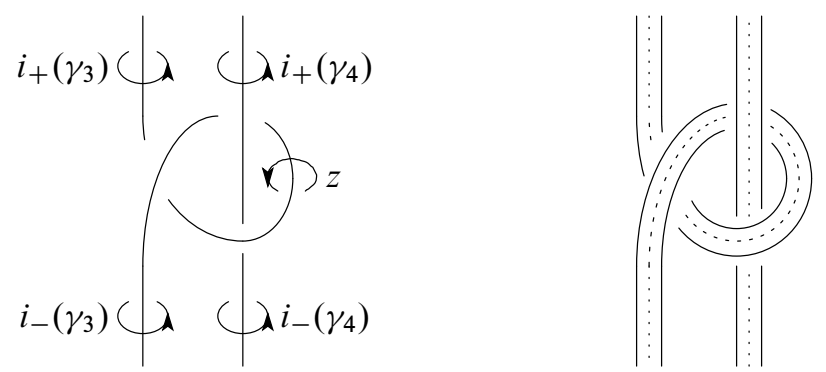

Figure 5: On the left, a 2-component pure string link $L$ and on the right, a tubular neighborhood of $L$

Then the presentation of $\pi_{1} M_{L}$ is given by

$$
\pi_{1} M_{L} \cong\left\langle\begin{array}{c|l}
i_{-}\left(\gamma_{1}\right), \ldots, i_{-}\left(\gamma_{4}\right) & \begin{array}{l}
i_{+}\left(\gamma_{1}\right) i_{-}\left(\gamma_{3}\right)^{-1} i_{+}\left(\gamma_{4}\right) i_{-}\left(\gamma_{1}\right)^{-1}, \\
z
\end{array} \\
\begin{array}{l}
\left.i_{+}\left(\gamma_{1}\right), i_{+}\left(\gamma_{3}\right)\right] i_{+}\left(\gamma_{2}\right) z i_{-}\left(\gamma_{2}\right)^{-1}\left[i_{-}\left(\gamma_{3}\right), i_{-}\left(\gamma_{1}\right)\right], \\
i_{+}\left(\gamma_{4}\right) i_{-}\left(\gamma_{3}\right) i_{+}\left(\gamma_{4}\right)^{-1} z^{-1}, \\
i_{+}\left(\gamma_{1}\right), \ldots, i_{+}\left(\gamma_{4}\right) \\
i_{-}\left(\gamma_{3}\right) i_{+}\left(\gamma_{3}\right)^{-1} i_{-}\left(\gamma_{3}\right)^{-1} z, \\
i_{-}\left(\gamma_{4}\right) z^{-1} i_{+}\left(\gamma_{4}\right)^{-1} z
\end{array}
\end{array}\right\},
$$

where we use the blackboard framing, namely the left dotted line in the right of Figure 5 is glued to $\left\{q_{1}\right\} \times I$ and the right one is glued to $\left\{q_{2}\right\} \times I$ in Figure 4 (with $g=2$ ). We now compute the Magnus matrix $r_{2}\left(M_{L}\right)$. We identify $N_{2}$ and $N_{2}\left(M_{L}\right)$ by using $i_{+}$. From the presentation, we have $z=i_{-}\left(\gamma_{3}\right)=\gamma_{3}, i_{-}\left(\gamma_{4}\right)=\gamma_{4}, i_{-}\left(\gamma_{2}\right)=\gamma_{2} \gamma_{3}$ 
and $i_{-}\left(\gamma_{1}\right)=\gamma_{1} \gamma_{3}^{-1} \gamma_{4}$ in $N_{2}$. Then

$$
\begin{aligned}
\left(\begin{array}{l}
A \\
B
\end{array}\right) & =\left(\begin{array}{ccccc}
-1 & \gamma_{3}^{-1}-1 & 0 & 0 & 0 \\
0 & 0 & 0 & 0 & 0 \\
-\gamma_{1}^{-1} \gamma_{3} & 1-\gamma_{1}^{-1} \gamma_{3} \gamma_{4}^{-1} & \gamma_{4}^{-1} & 1-\gamma_{3} & 0 \\
0 & 0 & 0 & 0 & 1 \\
0 & \gamma_{2}^{-1} & -1 & \gamma_{3} & \gamma_{3}-\gamma_{3} \gamma_{4}^{-1}
\end{array}\right), \\
C & =\left(\begin{array}{ccccc}
1 & 1-\gamma_{3}^{-1} & 0 & 0 & 0 \\
0 & 1 & 0 & 0 & 0 \\
0 & \gamma_{1}^{-1}-1 & 0 & -1 & 0 \\
\gamma_{1}^{-1} \gamma_{3} & 0 & 1-\gamma_{3}^{-1} & 0 & -\gamma_{3}
\end{array}\right)
\end{aligned}
$$

Hence the Magnus matrix

$$
r_{2}\left(M_{L}\right)=-C\left(\begin{array}{l}
A \\
B
\end{array}\right)^{-1}\left(\begin{array}{c}
I_{4} \\
0_{(1,4)}
\end{array}\right)
$$

is given by

$$
\left(\begin{array}{cccc}
1 & 0 & 0 & 0 \\
0 & 1 & 0 & 0 \\
\frac{-\gamma_{1}^{-1}}{\gamma_{3}^{-1}+\gamma_{4}^{-1}-1} & \frac{\gamma_{2}^{-1} \gamma_{3}^{-1} \gamma_{4}^{-1}-\gamma_{4}^{-1}+1}{\gamma_{3}^{-1}+\gamma_{4}^{-1}-1} & \frac{\gamma_{3}^{-1}}{\gamma_{3}^{-1}+\gamma_{4}^{-1}-1} & \frac{\gamma_{4}^{-1}\left(\gamma_{4}^{-1}-1\right)}{\gamma_{3}^{-1}+\gamma_{4}^{-1}-1} \\
\frac{\gamma_{1}^{-1} \gamma_{3} \gamma_{4}^{-1}}{\gamma_{3}^{-1}+\gamma_{4}^{-1}-1} & \frac{\left(1-\gamma_{3}^{-1}\right)\left(\gamma_{2}^{-1} \gamma_{3}^{-1}-\gamma_{2}^{-1}-1\right)}{\gamma_{3}^{-1}+\gamma_{4}^{-1}-1} \frac{\gamma_{3}^{-1}-1}{\gamma_{3}^{-1}+\gamma_{4}^{-1}-1} & \frac{-\gamma_{3}^{-1} \gamma_{4}^{-1}+\gamma_{3}^{-1}+2 \gamma_{4}^{-1}-1}{\gamma_{3}^{-1}+\gamma_{4}^{-1}-1}
\end{array}\right) .
$$

\section{Higher-order Alexander invariants and torsion-degree functions}

In this section, we first summarize the theory of higher-order Alexander invariants along the lines of Harvey's papers $[12 ; 13]$. For our use, we generalize them to functions of matrices called torsion-degree functions.

A group $\Gamma$ is poly-torsion-free-abelian (PTFA, for short) if $\Gamma$ has a normal series of finite length whose successive quotients are all torsion-free abelian. In particular, free nilpotent quotients $N_{k}$ are PTFA for all $k \geq 2$. Note that every subgroup of a PTFA group is also PTFA. For each PTFA group $\Gamma$, the group ring $\mathbb{Z} \Gamma$ is known to be an Ore domain, so that it can be embedded in the right field of fractions $\mathcal{K}_{\Gamma}:=\mathbb{Z} \Gamma(\mathbb{Z} \Gamma-\{0\})^{-1}$, 
which is a skew field. We refer to Cohn [6] and Passman [26] for localizations of noncommutative rings.

We will also use the following localizations of $\mathbb{Z} \Gamma$ placed between $\mathbb{Z} \Gamma$ and $\mathcal{K}_{\Gamma}$. Let $\psi: \Gamma \rightarrow \mathbb{Z}$ be an epimorphism. Then we have an exact sequence

$$
1 \longrightarrow\left(\Gamma^{\psi}:=\operatorname{Ker} \psi\right) \longrightarrow \Gamma \stackrel{\psi}{\longrightarrow} \mathbb{Z} \longrightarrow 1 \text {. }
$$

We take a splitting $\xi: \mathbb{Z} \rightarrow \Gamma$ and put $\tilde{t}:=\xi(1) \in \Gamma$. Since $\Gamma^{\psi}$ is again a PTFA group, $\mathbb{Z} \Gamma^{\psi}$ can be embedded in its right field of fractions $\mathcal{K}_{\Gamma^{\psi}}=\mathbb{Z} \Gamma^{\psi}\left(\mathbb{Z} \Gamma^{\psi}-\{0\}\right)^{-1}$. Moreover, we can also construct $\mathbb{Z} \Gamma\left(\mathbb{Z} \Gamma^{\psi}-\{0\}\right)^{-1}$. Then the splitting $\xi$ gives an isomorphism between $\mathbb{Z} \Gamma\left(\mathbb{Z} \Gamma^{\psi}-\{0\}\right)^{-1}$ and the skew Laurent polynomial ring $\mathcal{K}_{\Gamma^{\psi}}\left[t^{ \pm 1}\right]$, in which $a t=t\left(\widetilde{t}^{-1} a \tilde{t}\right)$ holds for each $a \in \mathcal{K}_{\Gamma^{\psi}} . \mathcal{K}_{\Gamma^{\psi}}\left[t^{ \pm 1}\right]$ is known to be a noncommutative right and left principal ideal domain. By definition, we have inclusions

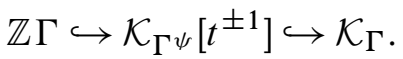

$\mathcal{K}_{\Gamma^{\psi}}\left[t^{ \pm 1}\right]$ and $\mathcal{K}_{\Gamma}$ are known to be flat $\mathbb{Z} \Gamma$-modules. On $\mathcal{K}_{\Gamma^{\psi}}\left[t^{ \pm 1}\right]$, we have a map deg ${ }^{\psi}: \mathcal{K}_{\Gamma \psi}\left[t^{ \pm 1}\right] \rightarrow \mathbb{Z}_{\geq 0} \cup\{\infty\}$ assigning to each polynomial its degree. We put

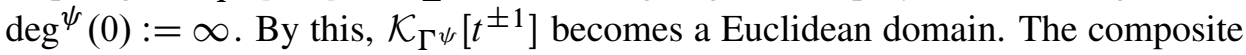

$$
\mathbb{Z} \Gamma\left(\mathbb{Z} \Gamma^{\psi}-\{0\}\right)^{-1} \stackrel{\cong}{\longrightarrow} \mathcal{K}_{\Gamma^{\psi}}\left[t^{ \pm 1}\right] \stackrel{\operatorname{deg}^{\psi}}{\longrightarrow} \mathbb{Z}_{\geq 0} \cup\{\infty\}
$$

does not depend on the choice of $\xi$.

Harvey's higher-order Alexander invariants [13] are defined as follows. Let $G$ be a finitely presentable group and let $\varphi: G \rightarrow \mathbb{Z}$ be an epimorphism. For a PTFA group $\Gamma$ and an epimorphism $\varphi_{\Gamma}: G \rightarrow \Gamma,\left(\varphi_{\Gamma}, \varphi\right)$ is called an admissible pair for $G$ if there exists an epimorphism $\psi: \Gamma \rightarrow \mathbb{Z}$ satisfying $\varphi=\psi \circ \varphi_{\Gamma}$. For each admissible pair $\left(\varphi_{\Gamma}, \varphi\right)$ for $G$, we regard $\mathcal{K}_{\Gamma^{\psi}}\left[t^{ \pm 1}\right]=\mathbb{Z} \Gamma\left(\mathbb{Z} \Gamma^{\psi}-\{0\}\right)^{-1}$ as a $\left(\mathbb{Z} G, \mathcal{K}_{\Gamma^{\psi}}\right)$ or $\left(\mathbb{Z} G, \mathcal{K}_{\Gamma^{\psi}}\left[t^{ \pm 1}\right]\right)$-module and we define the higher-order Alexander invariant for $\left(\varphi_{\Gamma}, \varphi\right)$ by

$$
\begin{aligned}
& \bar{\delta}_{\Gamma}^{\psi}(G)=\operatorname{dim}_{\mathcal{K}_{\Gamma \psi}}\left(H_{1}\left(G ; \mathcal{K}_{\Gamma^{\psi}}\left[t^{ \pm 1}\right]\right)\right) \in \mathbb{Z}_{\geq 0} \cup\{\infty\},
\end{aligned}
$$

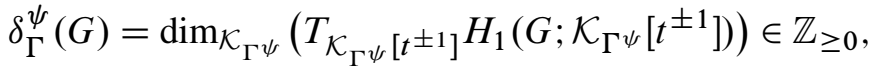

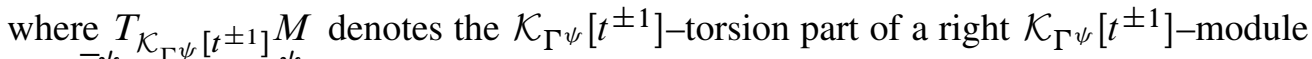
$M . \bar{\delta}_{\Gamma}^{\psi}(G)$ and $\delta_{\Gamma}^{\psi}(G)$ are called the $\Gamma$-degree and the refined $\Gamma$-degree respectively. (Our definition and notation are slightly different from those of [13], where the $\Gamma-$ degree is defined to be zero when it is $\infty$ in our definition.) Note that the right 
$\mathcal{K}_{\Gamma^{\psi}}\left[t^{ \pm 1}\right]$-module $H_{1}\left(G ; \mathcal{K}_{\Gamma^{\psi}}\left[t^{ \pm 1}\right]\right)$ can be decomposed into

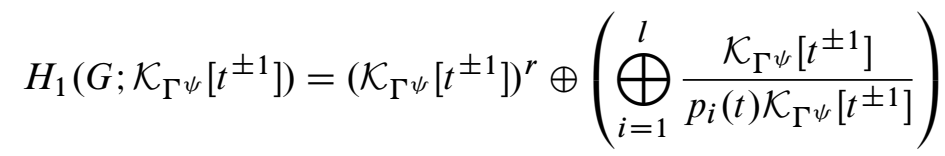

for some $r \in \mathbb{Z}_{\geq 0}$ and $p_{i}(t) \in \mathcal{K}_{\Gamma^{\psi}}\left[t^{ \pm 1}\right]$, then

$$
\begin{aligned}
& \bar{\delta}_{\Gamma}^{\psi}(G)= \begin{cases}\sum_{i=1}^{l} \operatorname{deg}^{\psi}\left(p_{i}(t)\right) & (r=0), \\
\infty & (r>0),\end{cases} \\
& \delta_{\Gamma}^{\psi}(G)=\sum_{i=1}^{l} \operatorname{deg}^{\psi}\left(p_{i}(t)\right) .
\end{aligned}
$$

For a connected space $X$ and an admissible pair $\left(\varphi_{\Gamma}, \varphi\right)$ for $\pi_{1} X$, we define $\bar{\delta}_{\Gamma}^{\psi}(X):=$ $\bar{\delta}_{\Gamma}^{\psi}\left(\pi_{1} X\right)$ and $\delta_{\Gamma}^{\psi}(X):=\delta_{\Gamma}^{\psi}\left(\pi_{1} X\right)$.

For a finitely presentable group $G$ and an admissible pair $\left(\varphi_{\Gamma}, \varphi\right)$ for $G$, the (refined) $\Gamma$-degree can be computed from any presentation matrix of the right $\mathcal{K}_{\Gamma^{\psi}}\left[t^{ \pm 1}\right]-$ module $H_{1}\left(G ; \mathcal{K}_{\Gamma^{\psi}}\left[t^{ \pm 1}\right]\right)$. Therefore we can consider it to be a function on the set $M\left(\mathcal{K}_{\Gamma^{\psi}}\left[t^{ \pm 1}\right]\right)$ of all matrices with entries in $\mathcal{K}_{\Gamma^{\psi}}\left[t^{ \pm 1}\right]$. Here we extend this function to $M\left(\mathcal{K}_{\Gamma}\right)$ as follows.

First, we extend $\operatorname{deg}^{\psi}: \mathcal{K}_{\Gamma^{\psi}}\left[t^{ \pm 1}\right] \rightarrow \mathbb{Z}_{\geq 0} \cup\{\infty\}$ to $\operatorname{deg}^{\psi}: \mathcal{K}_{\Gamma} \rightarrow \mathbb{Z} \cup\{\infty\}$ by setting $\operatorname{deg}^{\psi}\left(f g^{-1}\right)=\operatorname{deg}^{\psi}(f)-\operatorname{deg}^{\psi}(g)$ for $f \in \mathbb{Z} \Gamma, g \in \mathbb{Z} \Gamma-\{0\}$ (see for instance Cohn [6, Proposition 9.1.1]). It induces a group homomorphism $\operatorname{deg}^{\psi}:\left(\mathcal{K}_{\Gamma}^{\times}\right)_{\mathrm{ab}} \rightarrow \mathbb{Z}$, where $\left(\mathcal{K}_{\Gamma}^{\times}\right)_{\mathrm{ab}}$ is the abelianization of the multiplicative group $\mathcal{K}_{\Gamma}^{\times}=\mathcal{K}_{\Gamma}-\{0\}$.

Since $\mathcal{K}_{\Gamma}$ is a skew field, we have the Dieudonné determinant (see Rosenberg [27, Chapter 2])

$$
\text { det: } G L\left(\mathcal{K}_{\Gamma}\right) \longrightarrow\left(\mathcal{K}_{\Gamma}^{\times}\right)_{\mathrm{ab}}
$$

which is a homomorphism characterized by the following three properties:

(a) $\operatorname{det} I=1$.

(b) If $A^{\prime}$ is obtained by multiplying a row of a matrix $A \in G L\left(\mathcal{K}_{\Gamma}\right)$ by $a \in \mathcal{K}_{\Gamma}^{\times}$ from the left, then $\operatorname{det} A^{\prime}=a \cdot \operatorname{det} A$.

(c) If $A^{\prime}$ is obtained by adding to a row of a matrix $A$ a left $\mathcal{K}_{\Gamma}$-linear combination of other rows, then $\operatorname{det} A^{\prime}=\operatorname{det} A$.

It induces an isomorphism between $K_{1}\left(\mathcal{K}_{\Gamma}\right) \stackrel{\cong}{\rightrightarrows}\left(\mathcal{K}_{\Gamma}^{\times}\right)_{\mathrm{ab}}$.

The following lemma will be used in our generalization of Harvey's invariants. We denote by $M\left(m, n, \mathcal{K}_{\Gamma}\right)$ the set of all $m \times n$ matrices with entries in $\mathcal{K}_{\Gamma}$. 
Lemma 5.1 For $A \in M\left(m, n, \mathcal{K}_{\Gamma}\right)$ with $\operatorname{rank}_{\mathcal{K}_{\Gamma}} A=k$, let $U \in M\left(m-k, m, \mathcal{K}_{\Gamma}\right)$, $V \in M\left(n, n-k, \mathcal{K}_{\Gamma}\right)$ be matrices satisfying

$$
\begin{cases}U A=0, & \operatorname{rank}_{\mathcal{K}} U=m-k, \\ A V=0, & \operatorname{rank}_{\mathcal{K}_{\Gamma}} V=n-k\end{cases}
$$

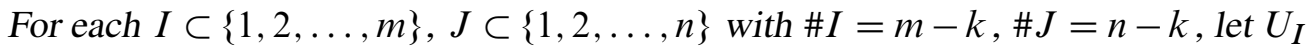
denote the square matrix defined by taking $i$-th columns from $U$ for all $i \in I$, and $V_{J}$ denote the one defined by taking $j$-th rows from $V$ for all $j \in J$. We also denote by $A_{I^{c} J^{c}}$ the one defined by taking $i$-th rows from $A$ for all $i \in I^{c}:=\{1,2, \ldots, m\}-I$ and then taking $j-$ th columns for all $j \in J^{c}:=\{1,2, \ldots, n\}-J$.

(1) If $U_{I}$ or $V_{J}$ is not invertible, then $A_{I^{c} J^{c}}$ is not invertible.

(2) Otherwise,

$$
\Delta(A ; U, V):=\operatorname{sgn}\left(I I^{c}\right) \operatorname{sgn}\left(J J^{c}\right) \frac{\operatorname{det} A_{I^{c} J^{c}}}{\operatorname{det} U_{I} \operatorname{det} V_{J}} \in\left(\mathcal{K}_{\Gamma}^{\times}\right)_{\mathrm{ab}}
$$

is independent of the choice of $I$ and $J$ such that $U_{I}, V_{J}$ are invertible, where $\operatorname{sgn}\left(I I^{c}\right) \in\{ \pm 1\}$ (resp. $\operatorname{sgn}\left(J J^{c}\right)$ ) is the signature of the juxtaposition of $I$ and $I^{c}$ (resp. $J$ and $J^{c}$ ), and we put $\operatorname{det} \varnothing:=1$.

(3) For $P_{1} \in G L\left(m, \mathcal{K}_{\Gamma}\right), P_{2} \in G L\left(n, \mathcal{K}_{\Gamma}\right), Q_{1} \in G L\left(m-k, \mathcal{K}_{\Gamma}\right)$ and $Q_{2} \in$ $G L\left(n-k, \mathcal{K}_{\Gamma}\right)$,

$$
\Delta\left(P_{1}^{-1} A P_{2}^{-1} ; Q_{1} U P_{1}, P_{2} V Q_{2}\right)=\frac{\Delta(A ; U, V)}{\operatorname{det} P_{1} \operatorname{det} P_{2} \operatorname{det} Q_{1} \operatorname{det} Q_{2}} .
$$

Proof (1) and (2) are deduced from easy observation using noncommutative linear algebra. To prove (3), it suffices to show in the cases where $P_{1}, P_{2}, Q_{1}, Q_{2}$ are matrices of elementary transformations, and it can be easily checked.

Remark 5.2 In the above situation, the sequence

$$
0 \longrightarrow \mathcal{K}_{\Gamma}^{n-k} \stackrel{V \cdot}{\longrightarrow} \mathcal{K}_{\Gamma}^{n} \stackrel{A \cdot}{\longrightarrow} \mathcal{K}_{\Gamma}^{m} \stackrel{U \cdot}{\longrightarrow} \mathcal{K}_{\Gamma}^{m-k} \longrightarrow 0
$$

is exact. By taking the standard basis for each vector space, we regard the sequence as a based acyclic chain complex. Then we can take its torsion (see Milnor [23] and Turaev [31] for generalities of torsions). This torsion coincides with $\Delta(A ; U, V)$ up to sign.

As seen in Lemma $5.1(3), \Delta(A ; U, V)$ does depend on $U$ and $V$. The following definition and lemma give special choices of $U$ and $V$. 
Definition 5.3 Let $A \in M\left(m, n, \mathcal{K}_{\Gamma}\right)$ with $\operatorname{rank}_{\mathcal{K}_{\Gamma}} A=k .(U, V)$ is said to be $\psi$-primitive for $A$ if

(1) $U, V$ have entries in $\mathcal{K}_{\Gamma^{\psi}}\left[t^{ \pm 1}\right]$,

(2) the row vectors $u_{1}, \ldots, u_{m-k} \in\left(\mathcal{K}_{\Gamma^{\psi}}\left[t^{ \pm 1}\right]\right)_{m}$ of $U$ generate $\operatorname{Ker}(\cdot A) \cap$ $\left(\mathcal{K}_{\Gamma^{\psi}}\left[t^{ \pm 1}\right]\right)_{m}$ in $\left(\mathcal{K}_{\Gamma}\right)_{m}$ as a left $\mathcal{K}_{\Gamma^{\psi}}\left[t^{ \pm 1}\right]$-module,

(3) the column vectors $v_{1}, \ldots, v_{n-k} \in\left(\mathcal{K}_{\Gamma^{\psi}}\left[t^{ \pm 1}\right]\right)^{n}$ of $V$ generate $\operatorname{Ker}(A \cdot) \cap$ $\left(\mathcal{K}_{\Gamma^{\psi}}\left[t^{ \pm 1}\right]\right)^{n}$ in $\left(\mathcal{K}_{\Gamma}\right)^{n}$ as a right $\mathcal{K}_{\Gamma^{\psi}}\left[t^{ \pm 1}\right]$-module.

Lemma 5.4 (1) There exists a pair $(U, V)$ which is $\psi$-primitive for $A$.

(2) Suppose $U \in M\left(m-k, m, \mathcal{K}_{\Gamma^{\psi}}\left[t^{ \pm 1}\right]\right)$ and $V \in M\left(n, n-k, \mathcal{K}_{\Gamma^{\psi}}\left[t^{ \pm 1}\right]\right)$ satisfy $U A=0$ and $A V=0 .(U, V)$ is $\psi$-primitive for $A$ if and only if there exist $\widetilde{P}_{1} \in$ $G L\left(m, \mathcal{K}_{\Gamma^{\psi}}\left[t^{ \pm 1}\right]\right)$ and $\widetilde{P}_{2} \in G L\left(n, \mathcal{K}_{\Gamma^{\psi}}\left[t^{ \pm 1}\right]\right)$ satisfying

$$
U \widetilde{P}_{1}=\left(0_{(m-k, k)} \quad I_{m-k}\right), \quad \widetilde{P}_{2} V=\left(0_{(n-k, k)} \quad I_{n-k}\right)^{T} .
$$

(3) Suppose $(U, V)$ and $\left(U^{\prime}, V^{\prime}\right)$ are $\psi$-primitive for $A$. Then there exist $P_{1} \in$ $G L\left(m, \mathcal{K}_{\Gamma^{\psi}}\left[t^{ \pm 1}\right]\right), P_{2} \in G L\left(n, \mathcal{K}_{\Gamma^{\psi}}\left[t^{ \pm 1}\right]\right), Q_{1} \in G L\left(m-k, \mathcal{K}_{\Gamma^{\psi}}\left[t^{ \pm 1}\right]\right)$ and $Q_{2} \in$ $G L\left(n-k, \mathcal{K}_{\Gamma^{\psi}}\left[t^{ \pm 1}\right]\right)$ such that

$$
U P_{1}=U^{\prime}, \quad P_{2} V=V^{\prime}, \quad Q_{1} U=U^{\prime}, \quad V Q_{2}=V^{\prime} .
$$

Proof We prove only for $V$. The proof for $U$ is similar.

(1) For right $\mathcal{K}_{\Gamma^{\psi}}\left[t^{ \pm 1}\right]$-homomorphisms

$$
\left(\mathcal{K}_{\Gamma \psi}\left[t^{ \pm 1}\right]\right)^{n} \stackrel{i}{\hookrightarrow} \mathcal{K}_{\Gamma}^{n} \stackrel{A \cdot}{\longrightarrow} \mathcal{K}_{\Gamma}^{m},
$$

$\operatorname{Ker}((A \cdot) \circ i)=\operatorname{Ker}(A \cdot) \cap\left(\mathcal{K}_{\Gamma^{\psi}}\left[t^{ \pm 1}\right]\right)^{n}$ is a right free $\mathcal{K}_{\Gamma^{\psi}}\left[t^{ \pm 1}\right]$-module of rank $n-k$. We take a basis $v_{1}, \ldots, v_{n-k}$ and put $V=\left(v_{1}, \ldots, v_{n-k}\right)$. Then $V$ satisfies the desired property.

(2) Suppose $V$ generates $\operatorname{Ker}(A \cdot) \cap\left(\mathcal{K}_{\Gamma^{\psi}}\left[t^{ \pm 1}\right]\right)^{n}$. The quotient module $\left(\mathcal{K}_{\Gamma^{\psi}}\left[t^{ \pm 1}\right]\right)^{n} /$ $\operatorname{Ker}((A \cdot) \circ i)$ is $\mathcal{K}_{\Gamma^{\psi}}\left[t^{ \pm 1}\right]$-torsion free, and hence $\mathcal{K}_{\Gamma^{\psi}}\left[t^{ \pm 1}\right]$-free. Taking a splitting, we have a direct sum decomposition $\left(\mathcal{K}_{\Gamma^{\psi}}\left[t^{ \pm 1}\right]\right)^{n} \cong\left(\left(\mathcal{K}_{\Gamma^{\psi}}\left[t^{ \pm 1}\right]\right)^{n} / \operatorname{Ker}((A \cdot) \circ i)\right) \oplus$ $\operatorname{Ker}((A \cdot) \circ i)$. We can extend $V$ to obtain a basis $\left(\widetilde{v_{1}}, \ldots, \widetilde{v_{k}}, V\right)$ for $\left(\mathcal{K}_{\Gamma^{\psi}}\left[t^{ \pm 1}\right]\right)^{n}$. Then $\widetilde{P}_{2}:=\left(\widetilde{v_{1}}, \ldots, \widetilde{v_{k}}, V\right)^{-1}$ satisfies $\widetilde{P}_{2} V=\left(0_{(n-k, k)} \quad I_{n-k}\right)^{T}$. The inverse is clear.

(3) The existence of $P_{2}$ follows immediately from (2). That of $Q_{2}$ is also clear since $V$ and $V^{\prime}$ are bases of the same right $\mathcal{K}_{\Gamma^{\psi}}\left[t^{ \pm 1}\right]$-module. 
Definition 5.5 Let $\Gamma$ be a PTFA group and let $\psi: \Gamma \rightarrow \mathbb{Z}$ be an epimorphism.

(1) The torsion-degree function $d_{\Gamma}^{\psi}: M\left(\mathcal{K}_{\Gamma}\right) \rightarrow \mathbb{Z}$ is defined by

$$
d_{\Gamma}^{\psi}(A):=\operatorname{deg}^{\psi}(\Delta(A ; U, V))
$$

for a pair $(U, V)$ which is $\psi$-primitive for $A$.

(2) The truncated torsion-degree function $\bar{d}_{\Gamma}^{\psi}: M\left(\mathcal{K}_{\Gamma}\right) \rightarrow \mathbb{Z} \cup\{\infty\}$ is defined by

$$
\bar{d}_{\Gamma}^{\psi}(A):= \begin{cases}d_{\Gamma}^{\psi}(A) & \text { if } \operatorname{rank} A \geq m-1, \\ \infty & \text { otherwise }\end{cases}
$$

for $A \in M\left(m, n, \mathcal{K}_{\Gamma}\right)$.

That $d_{\Gamma}^{\psi}$ and $\bar{d}_{\Gamma}^{\psi}$ are well-defined follows from Lemma 5.1 and Lemma 5.4 together with the following lemma.

Lemma 5.6 For any $P \in G L\left(\mathcal{K}_{\Gamma^{\psi}}\left[t^{ \pm 1}\right]\right)$ and $\psi$, we have $\operatorname{deg}^{\psi}(\operatorname{det} P)=0$.

Proof Since $\mathcal{K}_{\Gamma^{\psi}}\left[t^{ \pm 1}\right]$ is a Euclidean domain, every $P \in G L\left(\mathcal{K}_{\Gamma^{\psi}}\left[t^{ \pm 1}\right]\right)$ can be decomposed as products of elementary matrices and diagonal matrices in $G L\left(\mathcal{K}_{\Gamma^{\psi}}\left[t^{ \pm 1}\right]\right)$, each of which, say $Q$, satisfies $\operatorname{deg}^{\psi}(\operatorname{det} Q)=0$.

Example 5.7 (1) For $A \in G L\left(\mathcal{K}_{\Gamma}\right)$, we have $d_{\Gamma}^{\psi}(A)=\bar{d}_{\Gamma}^{\psi}(A)=\operatorname{deg}^{\psi}(\operatorname{det} A)$. In particular, $d_{\Gamma}^{\psi}(P)=\bar{d}_{\Gamma}^{\psi}(P)=0$ holds for any $P \in G L\left(\mathcal{K}_{\Gamma^{\psi}}\left[t^{ \pm 1}\right]\right.$ ) (hence, for any $P \in G L(\mathbb{Z} \Gamma))$ and $\psi$ by Lemma 5.6.

(2) Let $M$ be a finitely generated right $\mathcal{K}_{\Gamma^{\psi}}\left[t^{ \pm 1}\right]$-module, and let $A$ be a presentation matrix of $M$. Then we have $d_{\Gamma}^{\psi}(A)=\operatorname{dim}_{\mathcal{K}_{\Gamma} \psi}\left(T_{\mathcal{K}_{\Gamma} \psi}\left[t^{ \pm 1}\right] M\right)$. As for $\bar{d}_{\Gamma}^{\psi}(A)$, we can see that $\bar{d}_{\Gamma}^{\psi}(A) \in \mathbb{Z}$ if and only if the rank of the $\mathcal{K}_{\Gamma^{\psi}}\left[t^{ \pm 1}\right]$-free part of $M$ is less than 2 .

(3) Let $G$ be a finitely presentable group. We take a presentation $\left\langle x_{1}, \ldots, x_{l}\right|$ $\left.r_{1}, \ldots, r_{m}\right\rangle$ of $G$. For an admissible pair $\left(\varphi_{\Gamma}, \varphi\right)$, the matrix

$$
A:=\overline{\varphi_{\Gamma}\left(\frac{\partial r_{j}}{\partial x_{i}}\right)} \underbrace{}_{\substack{1 \leq i \leq l \\ 1 \leq j \leq m}}
$$

at $\mathcal{K}_{\Gamma^{\psi}}\left[t^{ \pm 1}\right]$ gives a presentation matrix of $H_{1}\left(G,\{1\} ; \mathcal{K}_{\Gamma^{\psi}}\left[t^{ \pm 1}\right]\right)$. Then Harvey's invariants are given by

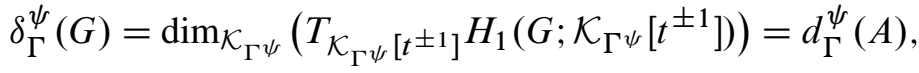

$$
\begin{aligned}
& \bar{\delta}_{\Gamma}^{\psi}(G)=\operatorname{dim}_{\mathcal{K}_{\Gamma \psi}}\left(H_{1}\left(G ; \mathcal{K}_{\Gamma^{\psi}}\left[t^{ \pm 1}\right]\right)\right)=\bar{d}_{\Gamma}^{\psi}(A),
\end{aligned}
$$


where the second equality of each case follows from the direct sum decomposition

$$
H_{1}\left(G,\{1\} ; \mathcal{K}_{\Gamma^{\psi}}\left[t^{ \pm 1}\right]\right) \cong H_{1}\left(G ; \mathcal{K}_{\Gamma^{\psi}}\left[t^{ \pm 1}\right]\right) \oplus \mathcal{K}_{\Gamma^{\psi}}\left[t^{ \pm 1}\right]
$$

shown by Harvey in [12, Proposition 5.6].

Remark 5.8 Friedl [8] gave an interpretation of Harvey's invariants by Reidemeister torsions. The definition of our truncated torsion-degree functions has some overlaps with his description. Note also that the restriction of $d_{\Gamma}^{\psi}$ or $\bar{d}_{\Gamma}^{\psi}$ to $G L\left(\mathcal{K}_{\Gamma}\right)$ coincides with the degree function in Friedl-Harvey [14, Section 2].

\section{Applications of torsion-degree functions to homology cylin- ders}

In this section, we study some invariants of homology cylinders arising from the Magnus representation and Reidemeister torsions by using torsion-degree functions associated with nilpotent quotients $N_{k}$ of $\pi_{1} \Sigma_{g, 1} . N_{k}$ is known to be a finitely generated torsion-free nilpotent group. In particular, it is PTFA.

Note that we can take a primitive element of $H^{1}\left(\Sigma_{g, 1}\right)$ instead of an epimorphism $N_{k} \rightarrow \mathbb{Z}$ to define a torsion-degree function since $\operatorname{Hom}\left(N_{k}, \mathbb{Z}\right)=H^{1}\left(N_{k}\right)=H^{1}\left(N_{2}\right)$ $=H^{1}\left(\Sigma_{g, 1}\right)$. We denote by $P H_{1}\left(\Sigma_{g, 1}\right)$ the set of primitive elements of $H^{1}\left(\Sigma_{g, 1}\right)$.

\subsection{The Magnus representation and torsion-degree functions}

First, we apply torsion-degree functions to the Magnus matrix. However, it turns out that the result is trivial.

Theorem 6.1 Let $M$ be a homology cylinder. For any $\psi \in P H^{1}\left(\Sigma_{g, 1}\right)$, the torsiondegree $d_{N_{k}}^{\psi}\left(r_{k}(M)\right)$ is always zero.

Proof By definition, $d_{N_{k}}^{\psi}$ is additive for products of invertible matrices, and invariant under taking the transpose and operating the involution. Moreover, it vanishes for matrices in $G L\left(\mathbb{Z} N_{k}\right)$ as seen in Example 5.7 (1). In [29], we show that there exists a matrix $\widetilde{J} \in G L\left(2 g, \mathbb{Z} N_{k}\right)$ satisfying the equality

$$
\overline{r_{k}(M)^{T}} \tilde{J} r_{k}(M)={ }^{\sigma_{k}(M)} \tilde{J} .
$$

By applying $d_{N_{k}}^{\psi}$ to it, we obtain $2 d_{N_{k}}^{\psi}\left(r_{k}(M)\right)=0$. This completes the proof. 
Example 6.2 Consider the homology cylinder $M_{L}$ in Example 4.4. $d_{N_{2}}^{\psi}\left(r_{2}\left(M_{L}\right)\right)$ is given by the degree of

$$
\operatorname{det} r_{2}\left(M_{L}\right)=\frac{\gamma_{3}+\gamma_{4}-1}{\gamma_{3} \gamma_{4}\left(\gamma_{3}^{-1}+\gamma_{4}^{-1}-1\right)}
$$

with respect to $\psi$. It is zero.

To extract some information from $r_{k}(M)$, we next apply torsion-degree functions to $I_{2 g}-r_{k}(M)$. The functions $d_{N_{k}}^{\psi}\left(I_{2 g}-r_{k}(\cdot)\right): \mathcal{C}_{g, 1} \rightarrow \mathbb{Z}$ and $\bar{d}_{N_{k}}^{\psi}\left(I_{2 g}-r_{k}(\cdot)\right): \mathcal{C}_{g, 1} \rightarrow$ $\mathbb{Z} \cup\{\infty\}$ factor through $\mathcal{H}_{g, 1}$ since $r_{k}$ does. Hence we can regard them as numerical invariants for $\mathcal{H}_{g, 1}$. In the rest of this subsection, we derive an explicit formula of $\bar{d}_{N_{k}}^{\psi}\left(I_{2 g}-r_{k}(\cdot)\right)$ under the assumption that $\left(M, i_{+}, i_{-}\right) \in \mathcal{C}_{g, 1}[k]$. Note that for every $\left(M, i_{+}, i_{-}\right) \in \mathcal{C}_{g, 1}[k]$, two inclusions $i_{+}$and $i_{-}$induce the same isomorphism $i_{+}=$ $i_{-}: N_{k} \cong N_{k}(M)$, so that we can naturally identify them. Under this identification, we have the following.

Lemma 6.3 Let $M$ be a homology cylinder belonging to $\mathcal{C}_{g, 1}[k]$.

(1) $\left(1-\gamma_{1}^{-1}, \ldots, 1-\gamma_{2 g}^{-1}\right)\left(I_{2 g}-r_{k}(M)\right)=0$.

(2) $\left(I_{2 g}-r_{k}(M)\right){\overline{\left(\frac{\partial \zeta}{\partial \gamma_{1}}, \ldots, \frac{\partial \zeta}{\partial \gamma_{2 g}}\right)}}^{T}=0$.

Proof We take an admissible presentation of $\pi_{1} M$ as in Definition 3.8. We also take the matrices $A, B, C \in M\left(\mathbb{Z} N_{k}\right)$ corresponding to it. For simplicity, we put

$$
\begin{aligned}
\overrightarrow{1-\vec{\gamma}} & :=\left(1-\gamma_{1}^{-1}, \ldots, 1-\gamma_{2 g}^{-1}\right), \\
\overrightarrow{1-\bar{z}} & :=\left(1-z_{1}^{-1}, \ldots, 1-z_{l}^{-1}\right), \\
\frac{\partial \zeta}{\partial \vec{\gamma}}: & =\left(\frac{\partial \zeta}{\partial \gamma_{1}}, \ldots, \frac{\partial \zeta}{\partial \gamma_{2 g}}\right) .
\end{aligned}
$$

(1) Using the fundamental formula of free calculus, we have

$$
\left(\begin{array}{lll}
1-\bar{\gamma} & \overrightarrow{1-\frac{z}{z}} & \overrightarrow{1-\bar{\gamma}}
\end{array}\right)\left(\begin{array}{l}
A \\
B \\
C
\end{array}\right)=0 .
$$

Then, by Proposition 3.9,

$$
(\overrightarrow{1-\vec{\gamma}} \quad \overrightarrow{1-\bar{z}})=-(\overrightarrow{1-\bar{\gamma}}) C\left(\begin{array}{l}
A \\
B
\end{array}\right)^{-1}=(\overrightarrow{1-\bar{\gamma}})\left(r_{k}(M) \quad Z\right)
$$

for some $Z \in M\left(2 g, l, \mathcal{K}_{N_{k}}\right)$. Our claim follows by taking their first $2 \mathrm{~g}$ columns. 
(2) Let $\tau_{\zeta} \in \mathcal{M}_{g, 1} \subset \mathcal{C}_{g, 1}$ be the Dehn twist along $\zeta$. It belongs to the center of $\mathcal{C}_{g, 1}$ and acts on $N_{k}$ by conjugation $x \mapsto \zeta^{-1} x \zeta$. Then

$$
\begin{aligned}
& r_{k}(M)=r_{k}\left(\tau_{\zeta}^{-1} M \tau_{\zeta}\right) \\
& =r_{k}\left(\tau_{\zeta}^{-1}\right) \cdot{ }^{\sigma_{k}\left(\tau_{\zeta}^{-1}\right)} r_{k}\left(M \tau_{\zeta}\right) \\
& ={ }^{\sigma_{k}\left(\tau_{\zeta}^{-1}\right)} r_{k}\left(\tau_{\zeta}\right)^{-1} \cdot{ }^{\sigma_{k}\left(\tau_{\zeta}^{-1}\right)}\left(r_{k}(M) \cdot{ }^{\sigma_{k}(M)} r_{k}\left(\tau_{\zeta}\right)\right) \\
& ={ }^{\sigma_{k}\left(\tau_{\zeta}^{-1}\right)}\left(r_{k}\left(\tau_{\zeta}\right)^{-1} \cdot r_{k}(M) \cdot r_{k}\left(\tau_{\zeta}\right)\right) \\
& =\left(\zeta I_{2 g}\right) \cdot r_{k}\left(\tau_{\zeta}\right)^{-1} \cdot r_{k}(M) \cdot r_{k}\left(\tau_{\zeta}\right) \cdot\left(\zeta^{-1} I_{2 g}\right)
\end{aligned}
$$

where the fourth equality follows from the fact that $M$ acts on $N_{k}$ trivially. On the other hand, it is easily checked that

$$
r_{k}\left(\tau_{\zeta}\right)=\left(I_{2 g}-\frac{\overline{\partial \zeta}}{\partial \vec{\gamma}} T(\overrightarrow{1-\bar{\gamma}})\right)\left(\zeta I_{2 g}\right)
$$

by using free differentials. Then

$$
\begin{aligned}
& r_{k}(M)=\left(I_{2 g}-\frac{\overrightarrow{\partial \zeta}}{\partial \vec{\gamma}} T(\overrightarrow{1-\bar{\gamma}})\right)^{-1} r_{k}(M)\left(I_{2 g}-\frac{\overrightarrow{\partial \zeta}}{\partial \vec{\gamma}}(\overrightarrow{1-\bar{\gamma}})\right) \\
\Longrightarrow & \left(I_{2 g}-\frac{\overrightarrow{\partial \zeta} T}{\partial \vec{\gamma}}(\overrightarrow{1-\vec{\gamma}})\right) r_{k}(M)=r_{k}(M)\left(I_{2 g}-\frac{\overrightarrow{\partial \zeta}}{\partial \vec{\gamma}}(\overrightarrow{1-\bar{\gamma}})\right) \\
\Longrightarrow & \frac{\overrightarrow{\partial \zeta} T}{\partial \vec{\gamma}}(\overrightarrow{1-\vec{\gamma}}) r_{k}(M)=r_{k}(M) \frac{\overrightarrow{\partial \zeta} T}{\partial \vec{\gamma}}(\overrightarrow{1-\vec{\gamma}}) .
\end{aligned}
$$

From (1), we see

$$
\frac{\overline{\partial \zeta}}{\partial \vec{\gamma}} T(\overrightarrow{1-\bar{\gamma}}) r_{k}(M)=\frac{\overline{\partial \zeta}}{\partial \vec{\gamma}} T(\overrightarrow{1-\bar{\gamma}})
$$

Comparing first columns, we have

$$
\frac{\overline{\partial \zeta}}{\partial \vec{\gamma}} T\left(1-\gamma_{1}^{-1}\right)=r_{k}(M) \frac{\overline{\partial \zeta} T}{\partial \vec{\gamma}}\left(1-\gamma_{1}^{-1}\right)
$$

(2) follows from this.

Proposition 6.4 If $M \in \mathcal{C}_{g, 1}[k]$ satisfies $\operatorname{rank}_{\mathcal{K}_{N_{k}}}\left(I_{2 g}-r_{k}(M)\right)=2 g-1$, then

$$
\bar{d}_{N_{k}}^{\psi}\left(I_{2 g}-r_{k}(M)\right)=\operatorname{deg}^{\psi}\left(\Delta\left(I_{2 g}-r_{k}(M) ; \overrightarrow{1-\bar{\gamma}}, \frac{\overrightarrow{\partial \zeta} T}{\partial \vec{\gamma}}\right)\right)
$$

Otherwise $\bar{d}_{N_{k}}^{\psi}\left(I_{2 g}-r_{k}(M)\right)=\infty$.

Proof By Lemma 6.3, the rank of $I_{2 g}-r_{k}(M)$ is at most $2 g-1$. The case where $\operatorname{rank}_{\mathcal{K}_{N_{k}}}\left(I_{2 g}-r_{k}(M)\right)<2 g-1$ is clear from Definition 5.5 (2). Suppose that 
$\operatorname{rank}_{\mathcal{K}_{N_{k}}}\left(I_{2 g}-r_{k}(M)\right)=2 g-1$. The task is to show that

$$
(\overrightarrow{1-\bar{\gamma}}) \text { and } \quad \frac{\overrightarrow{\partial \zeta}}{\partial \vec{\gamma}} T
$$

satisfy the conditions (2) and (3) of Definition 5.3 respectively. Suppose $(\overrightarrow{1-\bar{\gamma}})$ does not satisfy (2). Then $(\overrightarrow{1-\bar{\gamma}})$ can be written as $(\overrightarrow{1-\bar{\gamma}})=f v$, where $v \in\left(\mathcal{K}_{\left.N_{k}^{\psi}\left[t^{ \pm 1}\right]\right)_{2 g}}\right.$

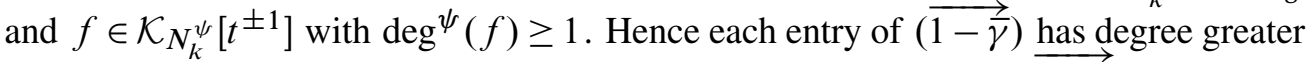
than 0 . When $A \in G L\left(\mathbb{Z} N_{k}\right)$, the same holds for each entry of $(\overrightarrow{1-\bar{\gamma}}) A$ which is nonzero. However, if we choose $f \in$ Aut $F_{2 g}$ such that $\psi\left(f\left(\gamma_{1}\right)\right)=0$, which does exist,

$$
\left(1-\gamma_{1}^{-1}, \ldots, 1-\gamma_{2 g}^{-1}\right) \overline{\left(\frac{\partial f\left(\gamma_{j}\right)}{\partial \gamma_{i}}\right)_{i, j}}=\left(1-f\left(\gamma_{1}\right)^{-1}, \ldots, 1-f\left(\gamma_{2 g}\right)^{-1}\right),
$$

a contradiction. Hence $(\overrightarrow{1-\vec{\gamma}})$ satisfies $(2)$. By a similar argument, we can show that

$$
\overline{\frac{\partial \zeta}{\partial \vec{\gamma}}} T
$$

satisfies (3), where we use $f \in$ Aut $F_{2 g}$ preserving $\zeta$ and the chain rule for free differentials (see Birman [2, Proposition 3.3]).

Note that $\bar{d}_{N_{k}}^{\psi}\left(I_{2 g}-r_{k}(M)\right)$ does not depend on the choice of a basis of $\pi_{1} \Sigma_{g, 1}$. This follows from the formulas in Proposition 3.10 and Lemma 5.1 (3). In Section 6.3.3, we will see some examples showing that this invariant is nontrivial at least in the case of $k=2$ for any $g \geq 1$.

\section{2 $N_{k}-$ torsion and torsion-degree functions}

Let $M=\left(M, i_{+}, i_{-}\right) \in \mathcal{C}_{g, 1}$. Since the relative complex $C_{*}\left(M, i_{+}\left(\Sigma_{g, 1}\right) ; \mathcal{K}_{N_{k}(M)}\right)$ obtained from any smooth triangulation of $\left(M, i_{+}\left(\Sigma_{g, 1}\right)\right)$ is acyclic by Lemma 3.1, we can consider its Reidemeister torsion $\tau\left(C_{*}\left(M, i_{+}\left(\Sigma_{g, 1}\right) ; \mathcal{K}_{N_{k}(M)}\right)\right)$.

Definition 6.5 The $N_{k}$-torsion of a homology cylinder $M=\left(M, i_{+}, i_{-}\right) \in \mathcal{C}_{g, 1}$ is given by

$$
\tau_{N_{k}}(M):={ }^{i-1} \tau\left(C_{*}\left(M, i_{+}\left(\Sigma_{g, 1}\right) ; \mathcal{K}_{N_{k}(M)}\right)\right) \in K_{1}\left(\mathcal{K}_{N_{k}}\right) /\left( \pm N_{k}\right) .
$$

Recall that Reidemeister torsions are invariant under subdivision of the cell complex $\left(M, i_{+}\left(\Sigma_{g, 1}\right)\right)$ and simple homotopy equivalence.

Now we consider $\tau_{N_{k}}(M)$ more closely. First we give a cell decomposition of $\partial M \cong$ $\Sigma_{g, 1} \cup\left(-\Sigma_{g, 1}\right)$ by pasting two copies of that of $\Sigma_{g, 1}$ in Figure 1. We denote by $R_{2 g}^{+}$the 
subcomplex $i_{+}\left(R_{2 g}\right)$. Take a triangulation of $\partial M$ by refining the cell decomposition, and extend it to one of $M$. Then

$$
\begin{aligned}
\tau\left(C_{*}\left(M, R_{2 g}^{+} ; \mathcal{K}_{N_{k}(M)}\right)\right)= & \tau\left(C_{*}\left(i_{+}\left(\Sigma_{g, 1}\right), R_{2 g}^{+} ; \mathcal{K}_{N_{k}(M)}\right)\right) \\
& \cdot \tau\left(C_{*}\left(M, i_{+}\left(\Sigma_{g, 1}\right) ; \mathcal{K}_{N_{k}(M)}\right)\right) \\
= & \tau\left(C_{*}\left(M, i_{+}\left(\Sigma_{g, 1}\right) ; \mathcal{K}_{N_{k}(M)}\right)\right)
\end{aligned}
$$

by the multiplicativity of torsions (see Milnor [23, Theorem 3.1], for example) and the fact that $i_{+}\left(\Sigma_{g, 1}\right)$ is simple homotopy equivalent to $R_{2 g}^{+}$.

Starting from a 3-simplex of $M$ facing the boundary, we can deform $M$ onto a 2dimensional subcomplex $M^{\prime}$ by a simple homotopy equivalence keeping the 1-skeleton of $M$ invariant. Then $\tau\left(C_{*}\left(M, R_{2 g}^{+} ; \mathcal{K}_{N_{k}(M)}\right)\right)=\tau\left(C_{*}\left(M^{\prime}, R_{2 g}^{+} ; \mathcal{K}_{N_{k}(M)}\right)\right)$. Next, we take a maximal tree $T$ of the 1 -skeleton of $M^{\prime}$ and collapse it to a point. This process also preserves the simple homotopy type of $\left(M^{\prime}, R_{2 g}^{+}\right)$, so that

$$
\tau\left(C_{*}\left(M^{\prime}, R_{2 g}^{+} ; \mathcal{K}_{N_{k}(M)}\right)\right)=\tau\left(C_{*}\left(M^{\prime} / T, R_{2 g}^{+} /\left(T \cap R_{2 g}^{+}\right) ; \mathcal{K}_{N_{k}(M)}\right)\right) .
$$

Consequently, $\tau_{N_{k}}(M)={ }^{i_{+}^{-1}} \tau\left(C_{*}\left(M^{\prime} / T, R_{2 g}^{+} /\left(T \cap R_{2 g}^{+}\right) ; \mathcal{K}_{N_{k}(M)}\right)\right) . M^{\prime} / T$ is a 2-dimensional cell complex with only one $0-$ cell, and $R_{2 g}^{+} /\left(T \cap R_{2 g}^{+}\right)$is a subcomplex consisting of one $0-$ cell and $2 g$ 1-cells. The pair $\left(M^{\prime} / T, R_{2 g}^{+} /\left(T \cap R_{2 g}^{+}\right)\right)$gives an admissible presentation

$$
\left\langle i_{-}\left(\gamma_{1}\right), \ldots, i_{-}\left(\gamma_{2 g}\right), z_{1}, \ldots, z_{l}, i_{+}\left(\gamma_{1}\right), \ldots, i_{+}\left(\gamma_{2 g}\right) \mid r_{1}, \ldots r_{2 g+l}\right\rangle
$$

of $\pi_{1} M$. For this presentation, we take the matrices $A, B, C \in M\left(\mathbb{Z} N_{k}\right)$ as in Section 3.2. Then

$$
\tau_{N_{k}}(M)={ }^{i_{+}^{-1}} \tau\left(C_{*}\left(M^{\prime} / T, R_{2 g}^{+} /\left(T \cap R_{2 g}^{+}\right) ; \mathcal{K}_{N_{k}(M)}\right)\right)={ }^{i_{+}^{-1}}\left(\begin{array}{l}
A \\
B
\end{array}\right) .
$$

Note that the matrix $\left(\begin{array}{l}A \\ B\end{array}\right)$ is a presentation matrix of

$$
H_{1}\left(M^{\prime} / T, R_{2 g}^{+} /\left(T \cap R_{2 g}^{+}\right) ; \mathbb{Z} N_{k}(M)\right)
$$

$\cong H_{1}\left(M, i_{+}\left(\Sigma_{g, 1}\right) ; \mathbb{Z} N_{k}(M)\right)$.

Since multiplying an element of $\pm N_{k}$ does not contribute to the degree, we have

$$
d_{N_{k}}^{\psi}\left(\tau_{N_{k}}(M)\right)=d_{N_{k}}^{\psi}\left({ }^{i_{+}^{-1}}\left(\begin{array}{l}
A \\
B
\end{array}\right)\right)=\operatorname{dim}_{\mathcal{K}_{N_{k}}^{\psi}} H_{1}\left(M, i_{+}\left(\Sigma_{g, 1}\right) ;\left(i_{+}^{-1}\right)^{*} \mathcal{K}_{\left.N_{k}^{\psi}\left[t^{ \pm 1}\right]\right)}\right.
$$

for each $\psi \in P H^{1}\left(\Sigma_{g, 1}\right)$. From this, we see that $d_{N_{k}}^{\psi}\left(\tau_{N_{k}}(M)\right)$ can be computed from any admissible presentation of $\pi_{1} M$ and that it does not depend on choices of cell decompositions of $\Sigma_{g, 1}$ and $M$. 
Proposition 6.6 Let $M_{1}=\left(M_{1}, i_{+}, i_{-}\right), M_{2}=\left(M_{2}, j_{+}, j_{-}\right) \in \mathcal{C}_{g, 1}$. Then

$$
d_{N_{k}}^{\psi}\left(\tau_{N_{k}}\left(M_{1} \cdot M_{2}\right)\right)=d_{N_{k}}^{\psi}\left(\tau_{N_{k}}\left(M_{1}\right)\right)+d_{N_{k}}^{\psi \circ \sigma_{2}\left(M_{1}\right)}\left(\tau_{N_{k}}\left(M_{2}\right)\right)
$$

holds for every $\psi \in P H^{1}\left(\Sigma_{g, 1}\right)$.

Proof We take an admissible presentation of $\pi_{1} M_{1}$ and the matrices $A_{M_{1}}, B_{M_{1}}, C_{M_{1}}$ corresponding to it. We denote this presentation by

$$
\pi_{1} M_{1} \cong\left\langle i_{-}(\vec{\gamma}), \vec{z}, i_{+}(\vec{\gamma}) \mid \vec{r}\right\rangle,
$$

for short. Similarly, we take an admissible presentation

$$
\pi_{1} M_{2} \cong\left\langle j_{-}(\vec{\gamma}), \vec{w}, j_{+}(\vec{\gamma}) \mid \vec{s}\right\rangle
$$

of $\pi_{1} M_{2}$ and the matrices $A_{M_{2}}, B_{M_{2}}, C_{M_{2}}$. Then we obtain an admissible presentation

$$
\pi_{1}\left(M_{1} \cdot M_{2}\right) \cong\left\langle j_{-}(\vec{\gamma}), \vec{w}, j_{+}(\vec{\gamma}), i_{-}(\vec{\gamma}), \vec{z}, i_{+}(\vec{\gamma}) \mid \vec{s}, j_{+}(\vec{\gamma}) i_{-}(\vec{\gamma})^{-1}, \vec{r}\right\rangle
$$

of $\pi_{1}\left(M_{1} \cdot M_{2}\right)$. The corresponding partial matrix $\left(\begin{array}{l}A_{M_{1} \cdot M_{2}} \\ B_{M_{1} \cdot M_{2}}\end{array}\right)$ at $\mathbb{Z} N_{k}\left(M_{1} \cdot M_{2}\right)$ is given by

$$
\left(\begin{array}{ccc}
{ }^{j} A_{M_{2}} & 0 & 0 \\
{ }^{j} B_{M_{2}} & 0 & 0 \\
{ }^{j} C_{M_{2}} & I_{2 g} & 0 \\
0 & -I_{2 g} & { }^{i} A_{M_{1}} \\
0 & 0 & { }^{i} B_{M_{1}}
\end{array}\right),
$$

where $i: M_{1} \rightarrow M_{1} \cdot M_{2}$ and $j: M_{2} \rightarrow M_{1} \cdot M_{2}$ are the natural inclusions. From this, we have

$$
\begin{aligned}
& d_{N_{k}}^{\psi}\left(\tau_{N_{k}}\left(M_{1} \cdot M_{2}\right)\right)=d_{N_{k}}^{\psi}\left({ }^{i \circ i_{+}^{-1}}\left(\begin{array}{l}
A_{M_{1} \cdot M_{2}} \\
B_{M_{1} \cdot M_{2}}
\end{array}\right)\right) \\
& =d_{N_{k}}^{\psi}\left({ }^{i_{+}^{-1}}\left(\begin{array}{l}
A_{M_{1}} \\
B_{M_{1}}
\end{array}\right)\right)+d_{N_{k}}^{\psi}\left({ }^{i_{+}^{-1} i^{-1} j}\left(\begin{array}{l}
A_{M_{2}} \\
B_{M_{2}}
\end{array}\right)\right) \\
& =d_{N_{k}}^{\psi}\left({ }^{i_{+}^{-1}}\left(\begin{array}{l}
A_{M_{1}} \\
B_{M_{1}}
\end{array}\right)\right)+d_{N_{k}}^{\psi}\left({ }^{\sigma_{k}\left(M_{1}\right) j_{+}^{-1}}\left(\begin{array}{l}
A_{M_{2}} \\
B_{M_{2}}
\end{array}\right)\right) \\
& =d_{N_{k}}^{\psi}\left(\tau_{N_{k}}\left(M_{1}\right)\right)+d_{N_{k}}^{\psi \circ \sigma_{2}\left(M_{1}\right)}\left(\tau_{N_{k}}\left(M_{2}\right)\right) .
\end{aligned}
$$

This completes the proof.

Remark 6.7 Proposition 6.6 can be seen as a generalization of Le Dimet [18, Proposition 1.11]. 


\subsection{Factorization formulas}

6.3.1 The $N_{k}$-degree for the closing of a homology cylinder For each $M=$ $\left(M, i_{+}, i_{-}\right) \in \mathcal{C}_{g, 1}$, we can construct a closed 3-manifold defined by

$$
C_{M}:=M /\left(i_{+}(x)=i_{-}(x)\right), \quad x \in \Sigma_{g, 1} .
$$

We call it the closing of $M$. It is easily seen that if $M \in \mathcal{C}_{g, 1}[k]$, we have the natural isomorphisms $N_{k}=N_{k}\left(\Sigma_{g, 1}\right) \cong N_{k}(M) \cong N_{k}\left(C_{M}\right)$. Here we identify these groups.

Theorem 6.8 Let $M=\left(M, i_{+}, i_{-}\right) \in \mathcal{C}_{g, 1}[k]$. For each $\psi \in P H^{1}\left(N_{k}\right)$, we have

$$
\bar{\delta}_{N_{k}}^{\psi}\left(C_{M}\right)=d_{N_{k}}^{\psi}\left(\tau_{N_{k}}(M)\right)+\bar{d}_{N_{k}}^{\psi}\left(I_{2 g}-r_{k}(M)\right) \in \mathbb{Z}_{\geq 0} \cup\{\infty\} .
$$

Proof Take an admissible presentation of $\pi_{1} M$ as in Definition 3.8, and construct the corresponding matrices $A, B, C \in M\left(\mathbb{Z} N_{k}\right)$.

Adding $2 g$ relations $i_{+}\left(\gamma_{j}\right)=i_{-}\left(\gamma_{j}\right)(j=1, \ldots, 2 g)$ and deleting the generators $i_{+}\left(\gamma_{j}\right)$ by using them, we obtain a presentation of $\pi_{1} C_{M}$. From this presentation, we have a presentation matrix $J_{C_{M}}$ of $H_{1}\left(C_{M}, p ; \mathbb{Z} N_{k}\right)$ given by

$$
J_{C_{M}}=\left(\begin{array}{c}
A+C \\
B
\end{array}\right)=\left(\begin{array}{cc}
I_{2 g}-r_{k}(M) & -Z \\
0_{(l, 2 g)} & I_{l}
\end{array}\right)\left(\begin{array}{l}
A \\
B
\end{array}\right)
$$

where $Z \in M\left(2 g, l, \mathcal{K}_{N_{k}}\right)$ is defined by the equality $\left(r_{k}(M) \quad Z\right)=-C\left({ }_{B}^{A}\right)^{-1}$ (see Proposition 3.9) so that $r_{k}(M) A+Z B=-C$ holds. Since $\left({ }_{B}^{A}\right)$ is invertible in $\mathcal{K}_{N_{k}}$,

$$
\begin{aligned}
& \operatorname{rank}_{\mathcal{K}_{N_{k}}} J_{C_{M}}=\operatorname{rank}_{\mathcal{K}_{N_{k}}}\left(\begin{array}{cc}
I_{2 g}-r_{k}(M) & -Z \\
0(l, 2 g) & I_{l}
\end{array}\right) \\
& =\operatorname{rank}_{\mathcal{K}_{N_{k}}}\left(I_{2 g}-r_{k}(M)\right)+l \leq 2 g+l-1 .
\end{aligned}
$$

Hence to show our claim, it suffices to prove the case where this value is just $2 g+l-1$ (see Definition 5.5 (2)).

By the fundamental formula of free calculus, we have

$$
(\overrightarrow{1-\bar{\gamma}} \overrightarrow{1-\bar{z}}) J_{C_{M}}=\left(1-\gamma_{1}^{-1}, \ldots, 1-\gamma_{2 g}^{-1}, 1-z_{1}^{-1}, \ldots, 1-z_{l}^{-1}\right) J_{C_{M}}=0 .
$$

On the other hand, we have

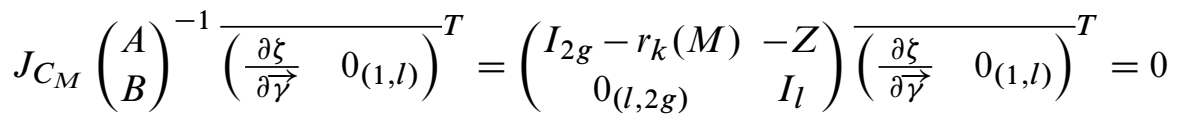


by Lemma 6.3 (2). Then we can define $\Delta\left(J_{C_{M}} ; \xi, \mu\right)$, where we put

$$
\begin{aligned}
\xi & :=\left(1-\gamma_{1}^{-1}, \ldots, 1-\gamma_{2 g}^{-1}, 1-z_{1}^{-1}, \ldots, 1-z_{l}^{-1}\right), \\
\mu & :=\left(\begin{array}{l}
A \\
B
\end{array}\right)^{-1} \frac{\left(\begin{array}{ll}
\frac{\partial \zeta}{\partial \vec{\gamma}} & \left.0_{(1, l)}\right)
\end{array}\right.}{T} .
\end{aligned}
$$

Lemma 6.9 $\mu$ belongs to $\left(\mathbb{Z} N_{k}\right)^{2 g+l}$.

Proof Recall that $\left(\begin{array}{c}A \\ B\end{array}\right)$ is a presentation matrix of $H_{1}\left(M, i_{+}\left(\Sigma_{g, 1}\right) ; \mathbb{Z} N_{k}\right)$, so that we have an exact sequence

$$
0 \longrightarrow\left(\mathbb{Z} N_{k}\right)^{2 g+l} \stackrel{\left(\begin{array}{l}
A \\
B
\end{array}\right) \cdot}{\longrightarrow}\left(\mathbb{Z} N_{k}\right)^{2 g+l} \longrightarrow H_{1}\left(M, i_{+}\left(\Sigma_{g, 1}\right) ; \mathbb{Z} N_{k}\right) \longrightarrow 0,
$$

where the injectivity of the second map follows since $H_{1}\left(M, i_{+}\left(\Sigma_{g, 1}\right) ; \mathcal{K}_{N_{k}}\right)=0$. Hence to prove the lemma, it suffices to show that in the third term $\left(\mathbb{Z} N_{k}\right)^{2 g+l}=$ $C_{1}\left(M, i_{+}\left(\Sigma_{g, 1}\right) ; \mathbb{Z} N_{k}\right)$,

$$
\overline{\left(\frac{\partial \zeta}{\partial \vec{\gamma}}\right.}_{\left.0_{(1, l)}\right)}^{T}
$$

is mapped to $0 \in H_{1}\left(M, i_{+}\left(\Sigma_{g, 1}\right) ; \mathbb{Z} N_{k}\right)$. In the exact sequence

$$
0 \rightarrow C_{1}\left(i_{+}\left(\Sigma_{g, 1}\right), p ; \mathbb{Z} N_{k}\right) \rightarrow C_{1}\left(M, p ; \mathbb{Z} N_{k}\right) \rightarrow C_{1}\left(M, i_{+}\left(\Sigma_{g, 1}\right) ; \mathbb{Z} N_{k}\right) \rightarrow 0
$$

the cycle

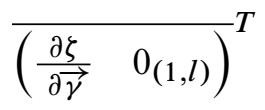

is attained by

$$
{\left.\overline{\left(\frac{\partial \zeta}{\partial \vec{\gamma}}\right.} \quad 0_{(1, l)} \quad 0_{(1,2 g)}\right)}^{T} \in C_{1}\left(M, p ; \mathbb{Z} N_{k}\right)=\left(\mathbb{Z} N_{k}\right)^{2 g+l} \oplus\left(\mathbb{Z} N_{k}\right)^{2 g}
$$

Then by observing the boundary corresponding to the relation

$$
\prod_{j=1}^{g}\left[i_{+}\left(\gamma_{j}\right), i_{+}\left(\gamma_{g+j}\right)\right]\left(\prod_{j=1}^{g}\left[i_{-}\left(\gamma_{j}\right), i_{-}\left(\gamma_{g+j}\right)\right]\right)^{-1}
$$

we see that

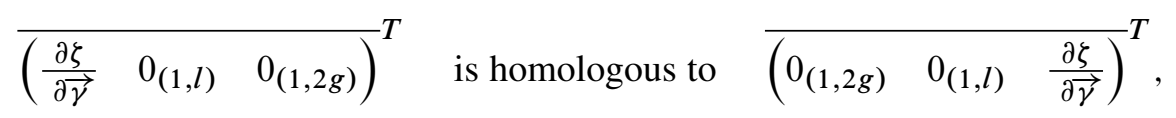

which comes from $C_{1}\left(i_{+}\left(\Sigma_{g, 1}\right), p ; \mathbb{Z} N_{k}\right)$. Our claim follows from this. 
Now we continue the proof of Theorem 6.8. We can show that $(\xi, \mu)$ is $\psi$-primitive for $J_{C_{M}}$ as in the proof of Proposition 6.4. Then we have

$$
\begin{aligned}
& \Delta\left(J_{C_{M}} ; \xi, \mu\right)=\Delta\left(J_{C_{M}} ;(\overrightarrow{1-\bar{\gamma}} \overrightarrow{1-\bar{z}}),\left(\begin{array}{l}
A \\
B
\end{array}\right)^{-1} \overrightarrow{\left(\begin{array}{ll}
\frac{\partial \zeta}{\partial \vec{\gamma}} & 0_{(1, l)}
\end{array}\right)}\right)
\end{aligned}
$$

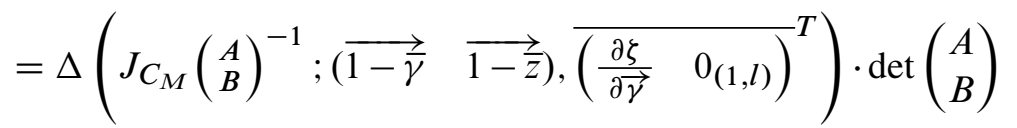

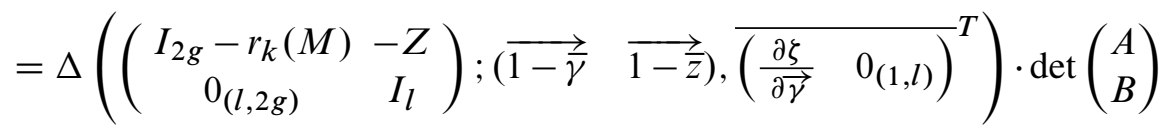

$$
\begin{aligned}
& =\Delta\left(I_{2 g}-r_{k}(M) ; \overrightarrow{1-\bar{\gamma}}, \frac{\overrightarrow{\partial \zeta} T}{\partial \vec{\gamma}}\right) \cdot \operatorname{det}\left(\begin{array}{l}
A \\
B
\end{array}\right) \text {. }
\end{aligned}
$$

From the above argument, we obtain

$$
\begin{aligned}
\bar{\delta}_{N_{k}}^{\psi}\left(C_{M}\right) & =\bar{d}_{N_{k}}^{\psi}\left(J_{C_{M}}\right)=\operatorname{deg}^{\psi}\left(\Delta\left(J_{C_{M}} ; \xi, \mu\right)\right) \\
& =\operatorname{deg}^{\psi}\left(\Delta\left(I_{2 g}-r_{k}(M) ; \overline{1-\bar{\gamma}}, \frac{\overline{\partial \zeta}}{\partial \vec{\gamma}} T\right)\right)+\operatorname{deg} \psi\left(\operatorname{det}\left(\begin{array}{l}
A \\
B
\end{array}\right)\right) \\
& =\bar{d}_{N_{k}}^{\psi}\left(I_{2 g}-r_{k}(M)\right)+d_{N_{k}}^{\psi}\left(\tau_{N_{k}}(M)\right) .
\end{aligned}
$$

This completes the proof of Theorem 6.8.

Remark 6.10 When $M \in \mathcal{C}_{g, 1}[k] \cap \mathcal{M}_{g, 1}, I_{2 g}-r_{k}(M)$ itself gives a presentation matrix of $H_{1}\left(C_{M}, p ; \mathbb{Z} N_{k}\right)$. Hence we have $\bar{\delta}_{N_{k}}^{\psi}\left(C_{M}\right)=\bar{d}_{N_{k}}^{\psi}\left(I_{2 g}-r_{k}(M)\right)$, and moreover $\delta_{N_{k}}^{\psi}\left(C_{M}\right)=d_{N_{k}}^{\psi}\left(I_{2 g}-r_{k}(M)\right)$ for this case.

6.3.2 The $N_{k, T}$-degree for the mapping torus of a homology cylinder Given $M=\left(M, i_{+}, i_{-}\right) \in \mathcal{C}_{g, 1}$, we have another method for obtaining a closed 3-manifold $T_{M}$ as follows. First we attach a $2-$ handle $I \times D^{2}$ to $M$ along $I \times i_{ \pm}\left(\partial \Sigma_{g, 1}\right)$ as in Figure 6. Let $M^{\prime}$ be the resulting 3-manifold. The markings $i_{+}$and $i_{-}$are naturally extended to embeddings $i_{+}^{\prime}, i_{-}^{\prime}: \Sigma_{g} \hookrightarrow \partial M^{\prime}$, where $\Sigma_{g}:=\Sigma_{g, 1} \cup D^{2}$ is an oriented closed surface of genus $g$.

Then we put

$$
T_{M}:=M^{\prime} /\left(i_{+}^{\prime}(x)=i_{-}^{\prime}(x)\right), \quad x \in \Sigma_{g}
$$

and call $T_{M}$ the mapping torus of $M$. Indeed, for $M_{\varphi} \in \mathcal{M}_{g, 1} \subset \mathcal{C}_{g, 1}$, the resulting manifold $T_{M_{\varphi}}$ is the usual mapping torus of $\varphi$ extended naturally to a mapping class of $\Sigma_{g}$. If we take an admissible presentation of $\pi_{1} M$ denoted by $\left\langle i_{-}(\vec{\gamma}), \vec{z}, i_{+}(\vec{\gamma}) \mid \vec{r}\right\rangle$ 


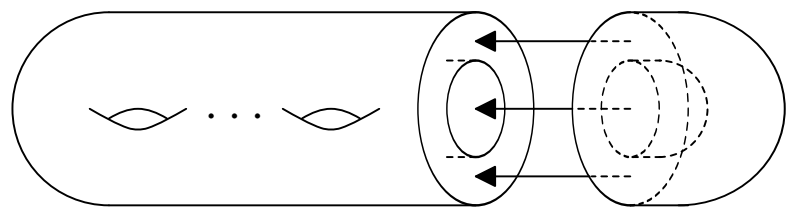

Figure 6: Attaching a 2-handle to $M$

briefly, then a presentation of $\pi_{1} T_{M}$ is given by

$\pi_{1} T_{M} \cong\left\langle i_{-}(\vec{\gamma}), \vec{z}, \lambda, i_{+}(\vec{\gamma}) \mid \vec{r}, \prod_{j=1}^{g}\left[i_{-}\left(\gamma_{j}\right), i_{-}\left(\gamma_{g+j}\right)\right], i_{-}(\vec{\gamma}) \lambda i_{+}(\vec{\gamma})^{-1} \lambda^{-1}\right\rangle$,

where $\lambda$ is the loop $I \times\{p\} \subset I \times D^{2} \subset T_{M}$. If $M \in \mathcal{C}_{g, 1}[k]$, we have natural isomorphisms $N_{k}\left(\Sigma_{g}\right) \cong N_{k}\left(M^{\prime}\right)$ and $N_{k}\left(T_{M}\right) \cong N_{k}\left(\Sigma_{g}\right) \times\langle\lambda\rangle$. Note that these groups are torsion-free nilpotent. We consider $N_{k}\left(\Sigma_{g}\right)$ to be a subgroup of $N_{k}\left(T_{M}\right)$. For simplicity, we denote $N_{k}\left(\Sigma_{g}\right)$ by $N_{k, 0}$ and $N_{k}\left(T_{M}\right)$ by $N_{k, T}$.

We can show that $H_{*}\left(M, i_{+}\left(\Sigma_{g, 1}\right) ; \mathcal{K}_{N_{k, T}}\right)=0$ (see Remark 3.2). Hence by a similar argument, the Magnus representation $r_{k, T}: \mathcal{C}_{g, 1} \rightarrow G L\left(2 g, \mathcal{K}_{N_{k, T}}\right)$ and the $N_{k, T^{-}}$ torsion

$$
\tau_{N_{k, T}}(M):=\tau\left(C_{*}\left(M, i_{+}\left(\Sigma_{g, 1}\right) ; \mathcal{K}_{N_{k, T}}\right)\right) \in K_{1}\left(\mathcal{K}_{N_{k, T}}\right) /\left( \pm N_{k, T}\right)
$$

are defined. Then we obtain the following factorization formula of the $N_{k, T}$-degree for the mapping torus of a homology cylinder.

Theorem 6.11 Let $M \in \mathcal{C}_{\underline{g}, 1}[k]$. For each primitive element $\psi \in H^{1}\left(N_{k, T}\right)=$ $H^{1}\left(T_{M}\right)$, the $N_{k, T}$-degree $\frac{\delta_{N_{k, T}}^{\psi}}{\psi}\left(T_{M}\right)$ is finite, and we have

$$
\begin{aligned}
\bar{\delta}_{N_{k, T}}^{\psi}\left(T_{M}\right) & =\delta_{N_{k, T}}^{\psi}\left(T_{M}\right) \\
& =d_{N_{k, T}}^{\psi}\left(\tau_{N_{k, T}}(M)\right)+d_{N_{k, T}}^{\psi}\left(I_{2 g}-\lambda r_{k, T}(M)\right)-2|\psi(\lambda)| .
\end{aligned}
$$

Proof The first assertion is a slight generalization of Harvey [12, Proposition 8.4], and we now follow the proof. Let $\psi \in H^{1}\left(T_{M}\right)$ be the Poincaré dual of the surface $i_{+}^{\prime}\left(\Sigma_{g}\right)=i_{-}^{\prime}\left(\Sigma_{g}\right)$. This gives an exact sequence $1 \rightarrow N_{k, 0} \rightarrow N_{k, T} \stackrel{\psi}{\rightarrow} \mathbb{Z} \rightarrow 1$. Then our claim is proved by showing that $\bar{\delta}_{N_{k, T}}^{\psi}\left(T_{M}\right)$ is finite for this $\psi$.

Let $\left(T_{M}\right)_{N_{k, T}}$ be the $N_{k, T}$-cover of $T_{M}$, and let $\left(T_{M}\right)_{\psi}$ be the $\mathbb{Z}$-cover of $T_{M}$ with respect to $\psi \cdot\left(T_{M}\right)_{\psi}$ is the product $\cdots M^{\prime} \cdot M^{\prime} \cdot M^{\prime} \cdots$ of countably many 
copies of $M^{\prime}$, and $\left(T_{M}\right)_{N_{k, T}}$ can be regarded as the $N_{k, 0}$-cover of $\left(T_{M}\right)_{\psi}$. Then

$$
\begin{aligned}
& H_{*}\left(T_{M} ; \mathcal{K}_{\left.N_{k, T}^{\psi}\left[t^{ \pm 1}\right]\right)}=H_{*}\left(C_{*}\left(\left(T_{M}\right)_{N_{k, T}}\right) \otimes_{N_{k, T}} \mathbb{Z} N_{k, T}\left(\mathbb{Z} N_{k, 0}-\{0\}\right)^{-1}\right)\right. \\
& \cong H_{*}\left(C_{*}\left(\left(T_{M}\right)_{N_{k, T}}\right) \otimes_{N_{k, 0}} \mathbb{Z} N_{k, 0}\left(\mathbb{Z} N_{k, 0}-\{0\}\right)^{-1}\right) \\
&=H_{*}\left(C_{*}\left(\left(\left(T_{M}\right)_{\psi}\right)_{N_{k, 0}}\right) \otimes_{N_{k, 0}} \mathcal{K}_{N_{k, T}^{\psi}}\right) \\
&=H_{*}\left(\left(T_{M}\right)_{\psi} ; \mathcal{K}_{N_{k, T}^{\psi}}\right) .
\end{aligned}
$$

Here we remark that the image of the composite $\pi_{1}\left(\left(T_{M}\right)_{\psi}\right) \rightarrow \pi_{1} T_{M} \rightarrow N_{k, T}$ is contained in $N_{k, 0}$. The same holds for the composite $\pi_{1} M^{\prime} \rightarrow \pi_{1} T_{M} \rightarrow N_{k, T}$. We also remark that $\mathcal{K}_{N_{k, T}^{\psi}}=\mathcal{K}_{N_{k, 0}}$.

We denote by $\Sigma$ again for a lift of $\Sigma \subset T_{M}$ on $\left(T_{M}\right)_{\psi}$. We divide $\left(T_{M}\right)_{\psi}$ at $\Sigma$, and obtain two parts $\left(T_{M}\right)_{\psi}^{+}$and $\left(T_{M}\right)_{\psi}^{-}$. Then $\left(T_{M}\right)_{\psi}^{ \pm}=\lim _{l}\left(M^{\prime}\right)^{l}$, and the inclusion $\Sigma \hookrightarrow\left(M^{\prime}\right)^{l}$ induces an isomorphism on homology. We can show that $H_{*}\left(\left(M^{\prime}\right)^{l}, \Sigma ; \mathcal{K}_{N_{k, T} \psi}\right)=0$ by the same way as mentioned in Lemma 3.1. Thus $H_{*}\left(\left(T_{M}\right)_{\psi}^{ \pm}, \Sigma ; \mathcal{K}_{N_{k, T} \psi}\right)=\underline{\lim }_{l} H_{*}\left(\left(M^{\prime}\right)^{l}, \Sigma ; \mathcal{K}_{N_{k, T} \psi}\right)=0$, and therefore $H_{*}\left(\left(T_{M}\right)_{\psi}, \Sigma ; \mathcal{K}_{N_{k, T} \psi}\right)=0$. This shows that

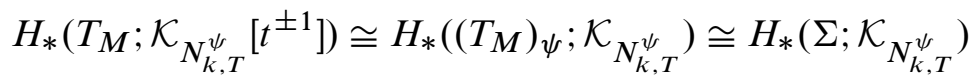

is a finite dimensional $\mathcal{K}_{N_{k, T}^{\psi}}$-vector space, so that $\bar{\delta}_{N_{k, T}}^{\psi}\left(T_{M}\right)$ is finite.

To show the second assertion, we take an admissible presentation of $\pi_{1} M$ and construct the matrices $A, B, C$ as before. We regard them as matrices with entries in $\mathbb{Z} N_{k, T}$. From the presentation, we have a presentation matrix $J_{T_{M}}$ of $H_{1}\left(T_{M}, p ; \mathbb{Z} N_{k, T}\right)$ given by

$$
J_{T_{M}}=\left(\begin{array}{ccc}
A & \overline{\frac{\partial \zeta}{\partial \vec{\gamma}} T} & I_{2 g} \\
B & 0_{(l, 1)} & 0_{(l, 2 g)} \\
0_{(1,2 g+l)} & 0 & -(\overline{1-\vec{\gamma}}) \\
C & 0_{(2 g, 1)} & -\lambda^{-1} I_{2 g}
\end{array}\right),
$$

where $\vec{\gamma}:=i_{+}(\vec{\gamma})=i_{-}(\vec{\gamma})$. We remark that $\lambda$ belongs to the center in $N_{k, T}$. As presentation matrices of $H_{1}\left(T_{M}, p ; \mathbb{Z} N_{k, T}\right)$, this matrix is equivalent to the square matrix

$$
J_{T_{M}}^{\prime}=\left(\begin{array}{cc}
A+\lambda C & \frac{\overrightarrow{\partial \zeta} T}{\partial \vec{\gamma}} \\
-(\overrightarrow{1-\vec{\gamma}}) \lambda C & 0
\end{array}\right) .
$$


By Proposition 3.9, Lemma 6.3 and the fundamental formula of free calculus, we have

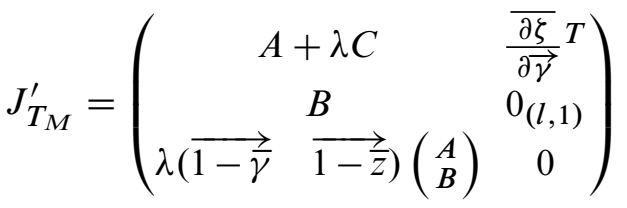

$$
\begin{aligned}
& =\left(\begin{array}{ccc}
I_{2 g}-\lambda r_{k, T}(M) & -\lambda Z & \frac{\overrightarrow{\partial \zeta} T}{\partial \vec{\gamma}} \\
0_{(l, 2 g)} & I_{l} & 0_{(l, 1)} \\
\lambda(\overrightarrow{1-\vec{\gamma}}) & \lambda(\overrightarrow{1-\vec{z}}) & 0
\end{array}\right)\left(\begin{array}{cc}
A & 0_{(2 g, 1)} \\
B & 0_{(l, 1)} \\
0_{(1,2 g+l)} & 1
\end{array}\right) .
\end{aligned}
$$

Note that

$$
\widetilde{\left(\begin{array}{l}
A \\
B
\end{array}\right)}:=\left(\begin{array}{cc}
A & 0_{(2 g, 1)} \\
B & 0_{(l, 1)} \\
0_{(1,2 g+l)} & 1
\end{array}\right)
$$

is invertible in $\mathcal{K}_{N_{k, T}}$. Then it is easily checked that

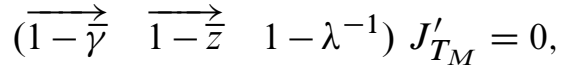

$$
\begin{aligned}
& J_{T_{M}}^{\prime}{\left(_{\left(\begin{array}{l}
A \\
B
\end{array}\right)}^{-1}\right.}^{-1} \frac{}{\left(\begin{array}{lll}
\frac{\partial \zeta}{\partial \vec{\gamma}} & 0_{(1, l)} & \lambda^{-1}-1
\end{array}\right)}=0 .
\end{aligned}
$$

We put

$$
\begin{aligned}
& \tilde{\xi}:=\left(\begin{array}{lll}
\overrightarrow{1-\bar{\gamma}} & \overrightarrow{1-\bar{z}} & 1-\lambda^{-1}
\end{array}\right)
\end{aligned}
$$

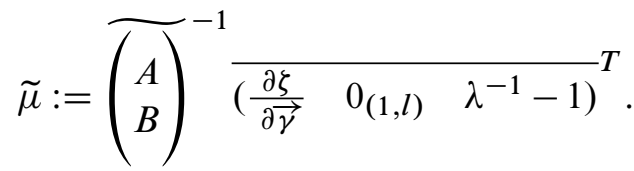

As in Proposition 6.4 and Lemma 6.9, we can show that $(\tilde{\xi}, \tilde{\mu})$ is $\psi$-primitive for $J_{T_{M}}^{\prime}$. Then

$$
\begin{aligned}
& \delta_{N_{k, T}}^{\psi}\left(T_{M}\right)=d_{N_{k, T}}^{\psi}\left(J_{T_{M}}^{\prime}\right)=\operatorname{deg}^{\psi}\left(\Delta\left(J_{T_{M}}^{\prime} ; \tilde{\xi}, \tilde{\mu}\right)\right) \\
& =\operatorname{deg} \psi\left(\Delta\left(J_{T_{M}}^{\prime} \widetilde{\left(\begin{array}{l}
A \\
B
\end{array}\right)}^{-1} ; \tilde{\xi}, \widetilde{\left(\begin{array}{l}
A \\
B
\end{array}\right)} \tilde{\mu}\right) \cdot \operatorname{det} \widetilde{\left(\begin{array}{l}
A \\
B
\end{array}\right)}\right)
\end{aligned}
$$

Algebraic 8 Geometric Topology, Volume 8 (2008) 


$$
\begin{aligned}
& =\operatorname{deg} \psi\left(\Delta\left(\left(\begin{array}{ccc}
I_{2 g}-\lambda r_{k, T}(M) & -\lambda Z & \frac{\overline{\partial \zeta}}{\partial \vec{\gamma}} \\
0_{(l, 2 g)} & I_{l} & 0_{(l, 1)} \\
\lambda(\overrightarrow{1-\vec{\gamma}}) & \lambda(\overrightarrow{1-\vec{z}}) & 0
\end{array}\right) ; \tilde{\xi}, \widetilde{\left(\begin{array}{c}
A \\
B
\end{array}\right) \tilde{\mu}}\right) \cdot \operatorname{det}\left(\begin{array}{l}
A \\
B
\end{array}\right)\right) \\
& =\operatorname{deg} \psi\left(\operatorname{det}\left(\begin{array}{cc}
I_{2 g}-\lambda r_{k, T}(M) & -\lambda Z \\
0_{(l, 2 g)} & I_{l}
\end{array}\right) \cdot \lambda^{-1}\left(1-\lambda^{-1}\right)^{-2} \cdot \operatorname{det} \widetilde{\left(\begin{array}{l}
A \\
B
\end{array}\right)}\right) \\
& =\operatorname{deg} \psi\left(\operatorname{det}\left(I_{2 g}-\lambda r_{k, T}(M)\right) \cdot \lambda^{-1}\left(1-\lambda^{-1}\right)^{-2} \cdot \operatorname{det} \widetilde{\left(\begin{array}{l}
A \\
B
\end{array}\right)}\right) \\
& =\operatorname{deg}^{\psi}\left(\operatorname{det}\left(I_{2 g}-\lambda r_{k, T}(M)\right)\right)+\operatorname{deg}{ }^{\psi}\left(\operatorname{det}\left(\tau_{N_{k, T}}(M)\right)\right)-2|\psi(\lambda)| \text {. }
\end{aligned}
$$

This completes the proof.

6.3.3 The case of $k=2$ (commutative case) Since $\mathbb{Z} N_{2}=\mathbb{Z} N_{2}\left(\Sigma_{g}\right)$ and $\mathcal{K}_{N_{2}}=$ $\mathcal{K}_{N_{2}\left(\Sigma_{g}\right)}$ are commutative, we can use the ordinary determinant for computations. Moreover, we can obtain some invariants before taking degrees. For example, define

$$
\Delta(M):=(-1)^{i+j} \frac{\operatorname{det}\left(\left(I_{2 g}-r_{2}(M)\right)_{(i, j)}\right)}{\left(1-\gamma_{i}^{-1}\right)\left(\frac{\partial \zeta}{\partial \gamma_{j}}\right)} \in \mathcal{K}_{N_{2}},
$$

where $A_{(i, j)}$ is the matrix obtained from a matrix $A$ by removing its $i$-th row and $j$-th column. $\Delta(M)$ is well-defined by Lemma 5.1. Note that this invariant is based on that for string links given in Kirk-Livingston-Wang [16, Definition 6.1], and we call it the Alexander rational function of $M$.

Theorem 6.12 Let $M \in \mathcal{C}_{g, 1}$ [2], and let $\Delta_{C_{M}}, \Delta_{T_{M}}$ be the Alexander polynomials of $C_{M}, T_{M}$, respectively. Then

$$
\begin{aligned}
\Delta_{C_{M}} & \doteq \overline{\tau_{N_{2}}(M) \cdot \Delta(M)}, \\
\Delta_{T_{M}} & \doteq \overline{\tau_{N_{2}}(M) \cdot \operatorname{det}\left(I_{2 g}-\lambda r_{2, T}(M)\right) \cdot\left(1-\lambda^{-1}\right)^{-2}},
\end{aligned}
$$

where $\doteq$ means that the left hand side coincides with the right hand side in $\mathcal{K}_{N_{2}}$ up to $\pm N_{2}$ or in $\mathcal{K}_{N_{2}\left(T_{M}\right)}$ up to $\pm N_{2}\left(T_{M}\right)$.

Proof We prove only the first assertion. The proof is almost the same as that for Theorem 6.8. We follow the notation used there. We may assume that $\operatorname{rank}_{\mathcal{K}_{N_{2}}} J_{C_{M}}=$ $\operatorname{rank}_{\mathcal{K}_{N_{2}}} r_{2}(M)+l=2 g+l-1$. 
By definition, $\Delta_{C_{M}}$ is the greatest common divisor of $\left\{\operatorname{det}{\overline{J_{C_{M}(i, j)}}}^{T}\right\}_{1 \leq i, j \leq 2 g+l}$. We show that it is nothing other than

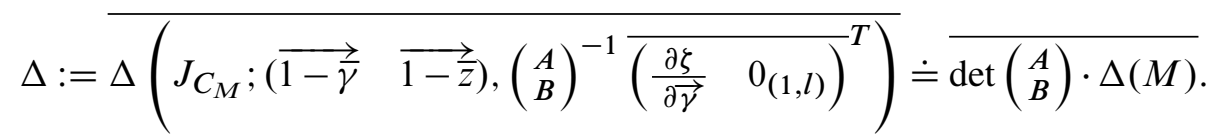

As seen in Lemma 6.9,

$$
\left(\begin{array}{l}
A \\
B
\end{array}\right)^{-1}{\left.\overline{\left(\frac{\partial \zeta}{\partial \vec{\gamma}}\right.} 0_{(1, l)}\right)}^{T}
$$

is a vector in $\left(\mathbb{Z} N_{2}\right)^{2 g+l}$. If $\Delta$ is in $\mathbb{Z} N_{2}$, it attains the greatest common divisor. To show it, suppose $\Delta=h_{1} / h_{2}$ where $h_{1} \in \mathbb{Z} N_{2}$ and $h_{2} \in \mathbb{Z} N_{2}-\{0\}$ are relatively prime. From the definition of $\Delta$, we have

$$
\frac{\left(1-\gamma_{i}^{-1}\right)\left(\overline{\frac{\partial \zeta}{\partial \gamma_{j}}}\right) h_{1}}{h_{2}}=(-1)^{i+j} \operatorname{det}{\overline{J_{C_{M}(i, j)}}}^{T} \in \mathbb{Z} N_{2} .
$$

Hence $h_{2}$ is a common divisor of each

$$
\left\{\left(1-\gamma_{i}^{-1}\right)\left(\overline{\frac{\partial \zeta}{\partial \gamma_{j}}}\right)\right\}_{i, j},
$$

and it is 1 . That is, $h_{2}$ is a unit in $\mathbb{Z} N_{2}$.

The determinant $\operatorname{det}\left({ }_{B}^{A}\right) \in \mathcal{K}_{N_{2}}$ (up to $\pm N_{2}$ ) does not depend on the choice of an admissible presentation, and it gives $\tau_{N_{2}}(M)$. Indeed the matrix $\left({ }_{B}^{A}\right)$ is a presentation matrix of $H_{1}\left(M, i_{+}\left(\Sigma_{g, 1}\right) ; \mathbb{Z} N_{2}\right)$ and its determinant gives a generator of the 0 -th elementary ideal, which is principal and invariant under Tietze transformations. This completes the proof.

Recall that the equality in Theorem 6.8 holds as elements of $\mathbb{Z} \cup\{\infty\}$. It is easily checked that $\bar{\delta}_{N_{k}}^{\psi}\left(C_{M}\right)=\infty$ if and only if $\bar{d}_{N_{k}}^{\psi}\left(I_{2 g}-r_{k}(M)\right)=\infty$, and this occurs when $H_{1}\left(C_{M} ; \mathcal{K}_{N_{k}^{\psi}}\left[t^{ \pm 1}\right]\right)$ has a nontrivial free part. The following are some examples of homology cylinders which have nontrivial (nonzero) Alexander rational functions. For these examples, the equality holds as elements of $\mathbb{Z}$. When $k \geq 3$, the computation becomes quite difficult in general.

Example 6.13 Assume that $g=1$. The Dehn twist $\tau_{\zeta} \in \mathcal{M}_{1,1}$ belongs to $\mathcal{C}_{1,1}$ [3]. A straightforward calculation shows that

$$
r_{2}\left(\tau_{\zeta}\right)=\left(\begin{array}{cc}
\gamma_{1}^{-1}+\gamma_{2}^{-1}-\gamma_{1}^{-1} \gamma_{2}^{-1} & -1+2 \gamma_{2}^{-1}-\gamma_{2}^{-2} \\
1-2 \gamma_{1}^{-1}+\gamma_{1}^{-2} & 2-\gamma_{1}^{-1}-\gamma_{2}^{-1}+\gamma_{1}^{-1} \gamma_{2}^{-1}
\end{array}\right)
$$

Then $\Delta\left(\tau_{\zeta}\right) \doteq 1 \in \mathbb{Z} N_{2}$, which is nontrivial. 
Example 6.14 Assume that $g \geq 2$. Let $\tau_{1}, \tau_{2}$ and $\tau_{3}$ be Dehn twists along simple closed curves $c_{1}, c_{2}$ and $c_{3}$ as in Figure 7 .

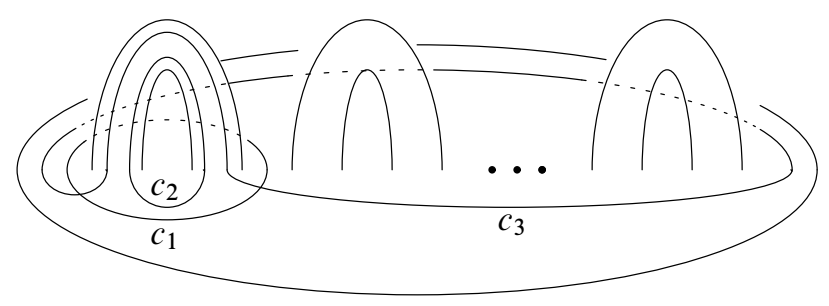

Figure 7

Then $\tau_{1} \tau_{2}^{-1}, \tau_{3} \in \mathcal{C}_{g, 1}[2]$. By a direct computation, we can check that $\Delta\left(\tau_{1} \tau_{2}^{-1} \cdot \tau_{3}\right) \doteq$ $-\left(\gamma_{1}-1\right)^{2 g-2}$, although $\Delta\left(\tau_{1} \tau_{2}^{-1}\right)=\Delta\left(\tau_{3}\right)=0$.

\section{4 $N_{k}$-torsions and Harvey's Realization Theorem}

By Proposition 6.6, the degree of the $N_{k}$-torsion gives a monoid homomorphism

$$
d_{N_{k}}^{\psi}\left(\tau_{N_{k}}(\cdot)\right): \mathcal{C}_{g, 1}[2] \longrightarrow \mathbb{Z}_{\geq 0}
$$

for each $\psi \in P H^{1}\left(\Sigma_{g, 1}\right)$ and an integer $k \geq 2$. To see some properties of these homomorphisms, including their nontriviality, we use a variant of Harvey's Realization Theorem in [12, Theorem 11.2] which gives a method for performing surgery on a compact orientable 3-manifold to obtain a homology cobordant one having distinct higher-order degrees.

Theorem 6.15 Let $M \in \mathcal{C}_{g, 1}$ be a homology cylinder. For each primitive element $x$ of $H_{1}\left(\Sigma_{g, 1}\right)$ and any integers $n \geq 2$ and $k \geq 1$, there exists a homology cylinder $M^{\prime}$ such that

(1) $M^{\prime}$ is homology cobordant to $M$,

(2) $d_{N_{l}}^{\psi}\left(\tau_{N_{l}}\left(M^{\prime}\right)\right)=d_{N_{l}}^{\psi}\left(\tau_{N_{l}}(M)\right)$ for $2 \leq l \leq n-1$,

(3) $d_{N_{n}}^{\psi}\left(\tau_{N_{n}}\left(M^{\prime}\right)\right) \geq d_{N_{n}}^{\psi}\left(\tau_{N_{n}}(M)\right)+k|p|$

for any $\psi \in P H^{1}\left(\Sigma_{g, 1}\right)$ satisfying $\psi(x)=p$. 
Proof The proof is based on Harvey's proof of Realization Theorem in [12, Theorem 11.2]. However, since we now use the lower central series instead of the rational derived series, we can shorten the argument.

We take a loop representing $x \in H_{1}\left(\Sigma_{g, 1}\right)$, and denote it by $x$ again. We also take a loop $\gamma$ whose homology class in $H_{1}\left(\Sigma_{g, 1}\right)$ is independent of $x$.

We attach a 1-handle to $M \times\{1\} \subset M \times I$, and then attach a 2-handle to obtain a 4-manifold $W$. Here the 2-handle are attached along the loop $\alpha\left[X_{n-1}, A_{k+1}\right]$, where $\alpha \in \pi_{1} M$ is a loop corresponding to the added 1-handle, and $X_{n-1}, A_{k+1} \in \pi_{1} M$ are inductively defined by

$$
\begin{array}{ll}
X_{1}=i_{+}(x), & X_{l}=\left[i_{+}(\gamma), X_{l-1}\right] \text { for } l \geq 2, \\
A_{1}=\alpha, & A_{l}=\left[i_{+}(x), A_{l-1}\right] \text { for } l \geq 2 .
\end{array}
$$

It is easily seen that $X_{l} \in \Gamma^{l}\left(\pi_{1} M\right)-\Gamma^{l+1}\left(\pi_{1} M\right) . M^{\prime}$ is defined as another part of $\partial W$, namely $\partial W=M \cup M^{\prime}$ and $M \cap M^{\prime}=\partial M=\partial M^{\prime}$. From the construction, we have $H_{*}(W, M)=0$. We also have $H_{*}\left(W, M^{\prime}\right)=0$ by using the Poincaré-Lefschetz duality and the universal coefficient theorem. Hence $\left(M^{\prime}, i_{+}, i_{-}\right) \in \mathcal{C}_{g, 1}$, and it is homology cobordant to $M$. Stallings' theorem shows that

$$
N_{l} \stackrel{i_{+}}{\longrightarrow} N_{l}(M) \rightarrow N_{l}(W) \leftarrow N_{l}\left(M^{\prime}\right) \stackrel{i_{+}}{\longleftarrow} N_{l}
$$

are all isomorphisms. Using them, we identify $N_{l}, N_{l}(M), N_{l}\left(M^{\prime}\right)$ and $N_{l}(W)$.

For simplicity, we put $K_{l}:=\mathcal{K}_{N_{l}^{\psi}}\left[t^{ \pm 1}\right]=\mathbb{Z} N_{l}\left(\mathbb{Z} N_{l}^{\psi}-\{0\}\right)^{-1}$. Recall that

$$
H_{*}\left(M, i_{+}\left(\Sigma_{g, 1}\right) ; \mathcal{K}_{N_{l}}\right)=H_{*}\left(M^{\prime}, i_{+}\left(\Sigma_{g, 1}\right) ; \mathcal{K}_{N_{l}}\right)=0
$$

as in Lemma 3.1. By the same proof, we have $H_{*}\left(W, i_{+}\left(\Sigma_{g, 1}\right) ; \mathcal{K}_{N_{l}}\right)=0$. Hence $H_{*}\left(M, i_{+}\left(\Sigma_{g, 1}\right) ; K_{l}\right), H_{*}\left(M^{\prime}, i_{+}\left(\Sigma_{g, 1}\right) ; K_{l}\right)$ and $H_{*}\left(W, i_{+}\left(\Sigma_{g, 1}\right) ; K_{l}\right)$ are all finite dimensional $\mathcal{K}_{N_{l}^{\psi}}$-vector spaces. As seen in Section 6.2,

$$
d_{N_{l}}^{\psi}\left(\tau_{N_{l}}(M)\right)=\operatorname{dim}_{\mathcal{K}_{N_{l}}} H_{1}\left(M, i_{+}\left(\Sigma_{g, 1}\right) ; K_{l}\right) .
$$

If we take an admissible presentation of $\pi_{1} M$ and the matrices $A, B \in \mathbb{Z} N_{k}$ as before, $\left(\begin{array}{l}A \\ B\end{array}\right)$ gives a presentation matrix of $H_{1}\left(M, i_{+}\left(\Sigma_{g, 1}\right) ; K_{l}\right)$. Then one of $H_{1}\left(W, i_{+}\left(\Sigma_{g, 1}\right) ; K_{l}\right)$ is given by

$$
\left(\begin{array}{cc}
A & * \\
0_{(1,2 g+l)} & \frac{*}{\partial \alpha\left[X_{n-1}, A_{k+1}\right]}
\end{array}\right),
$$

so that

$$
\operatorname{dim}_{\mathcal{K}_{N_{l}}^{\psi}} H_{1}\left(W, i_{+}\left(\Sigma_{g, 1}\right) ; K_{l}\right)=d_{N_{l}}^{\psi}\left(\tau_{N_{l}}(M)\right)+\operatorname{deg} \psi\left(\overline{\frac{\partial \alpha\left[X_{n-1}, A_{k+1}\right]}{\partial \alpha}}\right) .
$$


By a direct computation,

$$
\frac{\partial \alpha\left[X_{n-1}, A_{k+1}\right]}{\partial \alpha}=1+\alpha\left\{\left(1-X_{n-1} A_{k+1} X_{n-1}^{-1}\right) \frac{\partial X_{n-1}}{\partial \alpha}+\left(X_{n-1}-\left[X_{n-1}, A_{k+1}\right]\right) \frac{\partial A_{k+1}}{\partial \alpha}\right\} .
$$

When $2 \leq l \leq n-1$, we have $X_{n-1}=A_{k+1}=1 \in N_{l}$, so that $\frac{\partial \alpha\left[X_{n-1}, A_{k+1}\right]}{\partial \alpha}=1$, and $H_{1}\left(M, i_{+}\left(\Sigma_{g, 1}\right) ; K_{l}\right) \cong H_{1}\left(W, i_{+}\left(\Sigma_{g, 1}\right) ; K_{l}\right)$. When $l=n$, we have $X_{n-1} \neq$ $A_{k+1}=1 \in N_{l}$, so that

$$
\begin{aligned}
\frac{\partial \alpha\left[X_{n-1}, A_{k+1}\right]}{\partial \alpha} & =1+\left(X_{n-1}-1\right) \frac{\partial A_{k+1}}{\partial \alpha}=1+\left(X_{n-1}-1\right)\left(x-A_{k+1}\right) \frac{\partial A_{k}}{\partial \alpha} \\
& =\cdots=1+\left(X_{n-1}-1\right)\left(x-A_{k+1}\right)\left(x-A_{k}\right) \cdots\left(x-A_{2}\right),
\end{aligned}
$$

and $\quad \operatorname{deg}^{\psi}\left(\overline{\frac{\partial \alpha\left[X_{n-1}, A_{k+1}\right]}{\partial \alpha}}\right)=\operatorname{deg} \psi\left(\frac{\partial \alpha\left[X_{n-1}, A_{k+1}\right]}{\partial \alpha}\right)= \begin{cases}k|p| & (n \geq 3) \\ (k+1)|p| & (n=2) .\end{cases}$

In each case, $\operatorname{dim}_{\mathcal{K}_{N_{n}^{\psi}}} H_{1}\left(W, i_{+}\left(\Sigma_{g, 1}\right) ; K_{n}\right) \geq d_{N_{n}}^{\psi}\left(\tau_{N_{n}}(M)\right)+k|p|$.

By considering the dual handle decomposition, we see that $W$ is obtained from $M^{\prime} \times I$ by attaching a $2-$ handle and a 3 -handle. Hence $H_{1}\left(M^{\prime}, i_{+}\left(\Sigma_{g, 1}\right) ; K_{l}\right) \rightarrow$ $H_{1}\left(W, i_{+}\left(\Sigma_{g, 1}\right) ; K_{l}\right)$ is an epimorphism. In particular, when $l=n$,

$$
d_{N_{n}}^{\psi}\left(\tau_{N_{n}}\left(M^{\prime}\right)\right) \geq \operatorname{dim}_{\mathcal{K}_{N_{n}}} H_{1}\left(W, i_{+}\left(\Sigma_{g, 1}\right) ; K_{n}\right) \geq d_{N_{n}}^{\psi}\left(\tau_{N_{n}}(M)\right)+k|p| .
$$

It remains to proof that the map $H_{1}\left(M^{\prime}, i_{+}\left(\Sigma_{g, 1}\right) ; K_{l}\right) \rightarrow H_{1}\left(W, i_{+}\left(\Sigma_{g, 1}\right) ; K_{l}\right)$ is injective when $2 \leq l \leq n-1$. We now show that $H_{2}\left(W, M^{\prime} ; K_{l}\right)=0$. By the Poincaré-Lefschetz duality, $H_{2}\left(W, M^{\prime} ; K_{l}\right) \cong H^{2}\left(W, M ; K_{l}\right)$. On the other hand, it is easily checked that $H_{0}\left(W, M ; K_{l}\right)=H_{1}\left(W, M ; K_{l}\right)=H_{2}\left(W, M ; K_{l}\right)=0$. Then the universal coefficient spectral sequence (see Levine [19, Theorem 2.3]) shows our claim. Consequently, $H_{1}\left(M, i_{+}\left(\Sigma_{g, 1}\right) ; K_{l}\right) \cong H_{1}\left(W, i_{+}\left(\Sigma_{g, 1}\right) ; K_{l}\right) \cong$ $H_{1}\left(M^{\prime}, i_{+}\left(\Sigma_{g, 1}\right) ; K_{l}\right)$ and $d_{N_{l}}^{\psi}\left(\tau_{N_{l}}\left(M^{\prime}\right)\right)=d_{N_{l}}^{\psi}\left(\tau_{N_{l}}(M)\right)$. This completes the proof.

Corollary 6.16 For any $\psi \in P H^{1}\left(\Sigma_{g, 1}\right)$, the maps $\left\{d_{N_{k}}^{\psi}\left(\tau_{N_{k}}(\cdot)\right): \mathcal{C}_{g, 1}[2] \rightarrow \mathbb{Z}_{\geq 0}\right\}_{k \geq 2}$ are all nontrivial homomorphisms, and independent of each other. In particular, $\mathcal{C}_{g, 1}[2], \mathcal{C}_{g, 1}[3], \ldots, \operatorname{Ker}\left(\mathcal{C}_{g, 1} \rightarrow \mathcal{H}_{g, 1}\right)$ are not finitely generated as monoids.

In fact, we can show them by constructing homology cylinders that are homology cobordant to the unit $1_{\mathcal{C}_{g, 1}}$. Note that $d_{N_{k}}^{\psi}\left(\tau_{N_{k}}(M)\right)=0$ if $M \in \mathcal{M}_{g, 1}$ since $\Sigma_{g, 1} \times I$ is simple homotopy equivalent to $\Sigma_{g, 1}$ and hence $\tau_{N_{k}}(M)$ is trivial. 


\section{Application of torsion-degree functions to Aut $F_{n}^{\text {acy }}$}

In [28, Section 5], we defined the Magnus representation

$$
r_{k}: \text { Aut } F_{n}^{\text {acy }} \rightarrow G L\left(n, \mathcal{K}_{N_{k}\left(F_{n}\right)}\right)
$$

for the automorphism group of the acyclic closure (or HE-closure) $F_{n}^{\text {acy }}$ of $F_{n}$, where $F_{n}^{\text {acy }}$ is a completion of $F_{n}$ in a certain sense defined by Levine [20]. We also refer to Cha [3], where acyclic closures are called " $\mathbb{Z}$-closures".

The natural map $F_{n} \rightarrow F_{n}^{\text {acy }}$ is known to be injective and 2-connected. In particular, $N_{k}\left(F_{n}\right)=N_{k}\left(F_{n}^{\text {acy }}\right)$ by Stallings' theorem, and we denote it briefly by $N_{k}$ in this subsection. Aut $F_{n}^{\text {acy }}$ can be regarded as an enlargement of Aut $F_{n}$. Indeed we have the enlarged Dehn-Nielsen homomorphism $\sigma^{\text {acy }}: \mathcal{H}_{g, 1} \rightarrow$ Aut $F_{2 g}^{\text {acy }}$ extending the classical one $\sigma: \mathcal{M}_{g, 1} \hookrightarrow$ Aut $F_{2 g}$ [28, Section 6]. That is, we have the commutative diagram

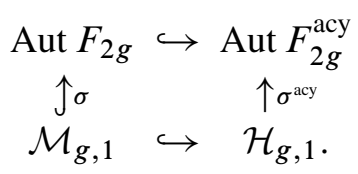

Note that $\sigma^{\text {acy }}$ is not injective. The Magnus representation for homology cylinders is nothing other than the composite

$$
\mathcal{H}_{g, 1} \stackrel{\sigma^{\text {acy }}}{\longrightarrow} \text { Aut } F_{2 g}^{\text {acy }} \stackrel{r_{k}}{\rightarrow} G L\left(2 g, \mathcal{K}_{N_{k}}\right) .
$$

We now consider the map $d_{N_{k}}^{\psi}$ or $r_{k}$ : Aut $F_{n}^{\text {acy }} \rightarrow \mathbb{Z}$ for $\psi \in P H^{1}\left(F_{n}\right)$, where $P H^{1}\left(F_{n}\right)$ denotes the set of primitive elements of $H^{1}\left(F_{n}\right)$. Since $d_{N_{k}}^{\psi}(A)=0$ for $A \in G L\left(\mathbb{Z} N_{k}\right)$ by Example 5.7 (1), it follows that $\left.d_{N_{k}}^{\psi} \circ r_{k}\right|_{\text {Aut } F_{n}}$ is trivial. When $n=2 g, d_{N_{k}}^{\psi} \circ$ $\left.r_{k}\right|_{\operatorname{Im} \sigma^{\text {acy }}}$ is also trivial as seen in Theorem 6.1. On the other hand, $d_{N_{k}}^{\psi} \circ r_{k}$ is actually nontrivial on Aut $F_{n}^{\text {acy }}$ as we will see below. Since $r_{k}$ is a crossed homomorphism, we have the following.

Proposition 7.1 For $f, g \in$ Aut $F_{n}^{\text {acy }}$ and $\psi \in P H^{1}\left(F_{n}\right)$, we have

$$
d_{N_{k}}^{\psi}\left(r_{k}(f g)\right)=d_{N_{k}}^{\psi}\left(r_{k}(f)\right)+d_{N_{k}}^{\psi \circ f}\left(r_{k}(g)\right) .
$$

In particular, if we restrict $d_{N_{k}}^{\psi} \circ r_{k}$ to

$$
\text { IAut } F_{n}^{\text {acy }}:=\operatorname{Ker}\left(\text { Aut } F_{n}^{\text {acy }} \rightarrow \text { Aut } N_{2} \cong G L(n, \mathbb{Z})\right),
$$

it becomes a homomorphism. 
Remark 7.2 As Aut $F_{n}^{\text {acy }}$ acts on $P H^{1}\left(F_{n}\right)$ from the right, it acts on $\operatorname{Map}\left(P H^{1}\left(F_{n}\right)\right.$, $\mathbb{Z})$ from the left. Regard $d_{N_{k}}\left(r_{k}(\cdot)\right)$ as a map Aut $F_{n}^{\text {acy }} \rightarrow \operatorname{Map}\left(P H^{1}\left(F_{n}\right), \mathbb{Z}\right)$. Proposition 7.1 shows that $d_{N_{k}}\left(r_{k}(\cdot)\right)$ is a 1-cocycle in $C^{1}\left(\right.$ Aut $\left.F_{n}^{\text {acy }}, \operatorname{Map}\left(P H^{1}\left(F_{n}\right), \mathbb{Z}\right)\right)$. We can see that it is nontrivial in $H^{1}$ (Aut $F_{n}^{\text {acy }}, \operatorname{Map}\left(P H^{1}\left(F_{n}\right), \mathbb{Z}\right)$ ) from the proof of Theorem 7.3 below.

IAut $F_{n}^{\text {acy }}$ can be regarded as an enlargement of IAut $F_{n}:=\operatorname{Ker}\left(\right.$ Aut $F_{n} \rightarrow$ Aut $N_{2}$ ). Nielsen [25] showed that IAut $F_{2} \cong F_{2}$. Magnus [22] gave a finite generating set of IAut $F_{n}$ for all $n \geq 3$. Andreadakis [1] (for $n=3$ ) and Cohen-Pakianathan, Farb and Kawazumi [15] (for all $n \geq 3$, independently), showed that the abelianization $H_{1}$ (IAut $\left.F_{n}\right)$ of IAut $F_{n}$ is isomorphic to $\operatorname{Hom}\left(F_{n}, \mathbb{Z}\right) \otimes \wedge^{2}\left(H_{1}\left(F_{n}\right)\right)$.

As for IAut $F_{n}^{\text {acy }}$, we have the following.

Theorem 7.3 For every $n \geq 2$, IAut $F_{n}^{\text {acy }}$ is not finitely generated. Moreover, the abelianization $H_{1}$ (IAut $F_{n}^{\text {acy }}$ ) of IAut $F_{n}^{\text {acy }}$ has infinite rank.

Proof Let $F_{n}=\left\langle x_{1}, x_{2}, \ldots, x_{n}\right\rangle$ and $\psi:=x_{1}^{*} \in P H^{1}\left(F_{n}\right)$. We show that the homomorphisms $\left\{d_{N_{k}}^{\psi}\left(r_{k}(\cdot)\right) \text { : IAut } F_{n}^{\text {acy }} \rightarrow \mathbb{Z}\right\}_{k \geq 2}$ are all nontrivial, and independent of each other.

Consider the endomorphism $f_{k}$ of $F_{n}$ given by

$$
f_{k}\left(x_{1}\right)=x_{1}\left[Y_{k-1}, Y_{k}\right], \quad f_{k}\left(x_{i}\right)=x_{i} \text { for } i \geq 2,
$$

where we define $Y_{1}=x_{1}$ and $Y_{l}=\left[x_{2}, Y_{l-1}\right]$ for $l \geq 2$. Since $f_{k}$ is 2-connected, it induces an automorphism of $F_{n}^{\text {acy }}$ [28, Section 4]. We denote it by $f_{k}$ again. It belongs to IAut $F_{n}^{\text {acy }}$. For such an automorphism, the Magnus matrix $r_{l}\left(f_{k}\right)$ can be computed by using free differentials. That is, we have

$$
r_{l}\left(f_{k}\right)=\left(\begin{array}{cc}
\frac{\overline{\frac{\partial f\left(x_{1}\right)}{\partial x_{1}}}}{\frac{\partial f\left(x_{1}\right)}{\partial x_{2}}} & 0_{(1, n-1)} \\
\frac{\vdots}{\frac{\partial f\left(x_{1}\right)}{\partial x_{n}}} & I_{n-1}
\end{array}\right)
$$

at $\mathbb{Z} N_{k}$. Then $d_{N_{l}}^{\psi}\left(r_{l}\left(f_{k}\right)\right)=\operatorname{deg} \psi\left(\operatorname{det}\left(r_{l}\left(f_{k}\right)\right)\right)=\operatorname{deg} \psi\left(\overline{\frac{\partial f\left(x_{1}\right)}{\partial x_{1}}}\right)=\operatorname{deg} \psi\left(\frac{\partial f\left(x_{1}\right)}{\partial x_{1}}\right)$. By a direct computation, we have

$$
\frac{\partial f\left(x_{1}\right)}{\partial x_{1}}=1+x_{1}\left\{\left(1-Y_{k-1} Y_{k} Y_{k-1}^{-1}\right) \frac{\partial Y_{k-1}}{\partial x_{1}}+\left(Y_{k-1}-\left[Y_{k-1}, Y_{k}\right]\right) \frac{\partial Y_{k}}{\partial x_{1}}\right\} .
$$

When $2 \leq l \leq k-1$, we have $Y_{k-1}=Y_{k}=1 \in N_{l}$, so that

$$
d_{N_{l}}^{\psi}\left(r_{l}\left(f_{k}\right)\right)=\operatorname{deg}^{\psi}\left(\frac{\partial f\left(x_{1}\right)}{\partial x_{1}}\right)=\operatorname{deg}^{\psi}(1)=0 .
$$


When $l=k$, we have $Y_{k-1} \neq 1 \in N_{k}$, so that

$$
\begin{aligned}
\frac{\partial f\left(x_{1}\right)}{\partial x_{1}}= & 1+x_{1}\left(Y_{k-1}-1\right) \frac{\partial Y_{k}}{\partial x_{1}}=1+x_{1}\left(Y_{k-1}-1\right)\left(x_{2}-Y_{k}\right) \frac{\partial Y_{k-1}}{\partial x_{1}} \\
= & \cdots=1+x_{1}\left(Y_{k-1}-1\right)\left(x_{2}-Y_{k}\right)\left(x_{2}-Y_{k-1}\right) \cdots\left(x_{2}-Y_{2}\right), \\
& d_{N_{k}}^{\psi}\left(r_{k}\left(f_{k}\right)\right)=\operatorname{deg}^{\psi}\left(\frac{\partial f\left(x_{1}\right)}{\partial x_{1}}\right)= \begin{cases}1 & (k \geq 3) \\
2 & (k=2) .\end{cases}
\end{aligned}
$$

and

Our claim follows from this.

Acknowledgements The author would like to express his gratitude to Professor Shigeyuki Morita for his encouragement and helpful suggestions. He also would like to thank Professor Masaaki Suzuki for valuable discussions and advice.

This research was supported by Grant-in-Aid for Scientific Research (No. 19840009), Ministry of Education, Science, Sports and Technology and by 21 st century COE program at Graduate School of Mathematical Sciences, The University of Tokyo.

Finally, the author would like to thank the referee for his/her careful reading of the manuscript and helpful suggestions.

\section{References}

[1] S Andreadakis, On the automorphisms of free groups and free nilpotent groups, Proc. London Math. Soc. (3) 15 (1965) 239-268 MR0188307

[2] J S Birman, Braids, links, and mapping class groups, Annals of Math. Studies 82, Princeton University Press (1974) MR0375281

[3] J Cha, Injectivity theorems and algebraic closures of groups with coefficients arXiv: math.GT/0609408

[4] T D Cochran, Noncommutative knot theory, Algebr. Geom. Topol. 4 (2004) 347-398 MR2077670

[5] T D Cochran, KE Orr, P Teichner, Knot concordance, Whitney towers and $L^{2}-$ signatures, Ann. of Math. (2) 157 (2003) 433-519 MR1973052

[6] P M Cohn, Skew fields. Theory of general division rings, Encyclopedia of Math. and its Applications 57, Cambridge University Press (1995) MR1349108

[7] R H Fox, Free differential calculus. II. The isomorphism problem of groups, Ann. of Math. (2) 59 (1954) 196-210 MR0062125

[8] S Friedl, Reidemeister torsion, the Thurston norm and Harvey's invariants, Pacific J. Math. 230 (2007) 271-296 MR2309160

Algebraic 8 Geometric Topology, Volume 8 (2008) 
[9] S Garoufalidis, J Levine, Tree-level invariants of three-manifolds, Massey products and the Johnson homomorphism, from: "Graphs and patterns in mathematics and theoretical physics”, Proc. Sympos. Pure Math. 73, Amer. Math. Soc. (2005) 173-203 MR2131016

[10] M Goussarov, Finite type invariants and $n$-equivalence of 3-manifolds, C. R. Acad. Sci. Paris Sér. I Math. 329 (1999) 517-522 MR1715131

[11] K Habiro, Claspers and finite type invariants of links, Geom. Topol. 4 (2000) 1-83 MR1735632

[12] S L Harvey, Higher-order polynomial invariants of 3-manifolds giving lower bounds for the Thurston norm, Topology 44 (2005) 895-945 MR2153977

[13] SL Harvey, Monotonicity of degrees of generalized Alexander polynomials of groups and 3-manifolds, Math. Proc. Cambridge Philos. Soc. 140 (2006) 431-450 MR2225642

[14] S L Harvey, S Friedl, Non-commutative multivariable Reidemester torsion and the Thurston norm, Algebr. Geom. Topol. 7 (2007) 755-777 MR2308963

[15] N Kawazumi, Cohomological aspects of Magnus expansions arXiv: math.GT/0505497

[16] P Kirk, C Livingston, Z Wang, The Gassner representation for string links, Commun. Contemp. Math. 3 (2001) 87-136 MR1820015

[17] J-Y Le Dimet, Enlacements d'intervalles et représentation de Gassner, Comment. Math. Helv. 67 (1992) 306-315 MR1161287

[18] J-Y Le Dimet, Enlacements d'intervalles et torsion de Whitehead, Bull. Soc. Math. France 129 (2001) 215-235 MR1871296

[19] J Levine, Knot modules. I, Trans. Amer. Math. Soc. 229 (1977) 1-50 MR0461518

[20] J Levine, Algebraic closure of groups, from: "Combinatorial group theory (College Park, MD, 1988)”, Contemp. Math. 109, Amer. Math. Soc. (1990) 99-105 MR1076380

[21] J Levine, Homology cylinders: an enlargement of the mapping class group, Algebr. Geom. Topol. 1 (2001) 243-270 MR1823501

[22] W Magnus, Über n-dimensionale Gittertransformationen, Acta Math. 64 (1935) 353367 MR1555401

[23] J Milnor, Whitehead torsion, Bull. Amer. Math. Soc. 72 (1966) 358-426 MR0196736

[24] S Morita, Abelian quotients of subgroups of the mapping class group of surfaces, Duke Math. J. 70 (1993) 699-726 MR1224104

[25] J Nielsen, Die Isomorphismen der allgemeinen, unendlichen Gruppe mit zwei Erzeugenden, Math. Ann. 78 (1964) 385-397 MR1511907

[26] D S Passman, The algebraic structure of group rings, Pure and Applied Mathematics, Wiley-Interscience, New York (1977) MR470211 
[27] J Rosenberg, Algebraic K-theory and its applications, Graduate Texts in Math. 147, Springer, New York (1994) MR1282290

[28] T Sakasai, Homology cylinders and the acyclic closure of a free group, Algebr. Geom. Topol. 6 (2006) 603-631 MR2220691

[29] T Sakasai, The symplecticity of the Magnus representation for homology cobordisms of surfaces, Bull. Austral. Math. Soc. 76 (2007) 421-431 MR2374173

[30] J Stallings, Homology and central series of groups, J. Algebra 2 (1965) 170-181 MR0175956

[31] V Turaev, Introduction to combinatorial torsions, Lectures in Math. ETH Zürich, Birkhäuser Verlag, Basel (2001) MR1809561Notes taken by Felix Schlenk

Graduate School of Mathematical Sciences, The University of Tokyo

3-8-1 Komaba, Meguro, Tokyo, 153-8914, Japan

sakasai@ms . u-tokyo.ac.jp

http://www.ms.u-tokyo.ac.jp/ sakasai/

Received: 30 November 2006

Algebraic $8 \mathcal{G}$ Geometric Topology, Volume 8 (2008) 